\title{
Joint Radar-Communication Relying on Spread Spectrum: A Hybrid Chaotic Sequence Design
}

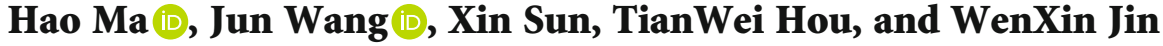 \\ School of Electronic and Information Engineering, Beijing Jiaotong University, Beijing, China \\ Correspondence should be addressed to Hao Ma; 16111020@bjtu.edu.cn and Jun Wang; wangjun1@bjtu.edu.cn
}

Received 19 April 2020; Revised 12 October 2020; Accepted 9 November 2020; Published 4 December 2020

Academic Editor: Gianluigi Ferrari

Copyright $\odot 2020$ Hao Ma et al. This is an open access article distributed under the Creative Commons Attribution License, which permits unrestricted use, distribution, and reproduction in any medium, provided the original work is properly cited.

\begin{abstract}
This paper investigates a novel design for joint radar-communication (JRC) waveform, which is capable of improving the network's performance by solving the phase shift mutation of the Chirp-BPSK waveform. We aim for jointly designing chirp waveform and improving Binary Phase Shift Keying (BPSK), as well as the hybrid chaotic spread spectrum code (CSSC). As a benefit, the JRC waveform can be successfully decoded, and the accumulation of phase shift can be suppressed by hybrid CSSC. The detailed deduction on the short-time Fourier transform (STFT) of the JRC waveform is adopted to analyse the complicated AF characters. Our analytical results demonstrate that the proposed JRC signal has superior spectrum performance, ambiguity function (AF), and transmission rate than conventional Chirp and Chirp-BPSK waveform. Numerical results are provided to confirm that (i) the proposed waveform is capable of providing better communication and radar performance; (ii) the performance of $\mathrm{AF}$ is not affected by the transmission rate for the proposed waveform.
\end{abstract}

\section{Introduction}

In recent years, the increasing congestion of spectrum resource cannot meet the ever-growing bandwidth requirements for communication and radar, for the fields of disaster monitoring, intelligent transportation, remote sensing, military confrontation, and so on $[1,2]$. Although in radio frequency (RF) front-end architectures, both the radar and communication become similar, operating radar and communication simultaneously cause a series of problems such as the number of antennas, the energy consumption, and electromagnetic signal interference. So the concept of joint radar-communication (JRC) is proposed in the recent few decades, which enables radar and wireless communication users working in the same frequency band, sharing the same antenna resources, implementing radar sensing, and communication transmission synchronously [3-8]. A typical JRC is illustrated in Figure 1, it is shown that the JRC signal is sent from the radar transmitter, and the communication and radar are operated simultaneously. The radar wave is propagating up and down, while the communication wave is transmitting through a one-way path.
Owing to strictly finite radio spectrum resource, the JRC has gained a strong interest in waveform designing, information embedding, coexistence algorithm, and so on. Furthermore, the suitable integration waveform is the research hotspots of JRC in recent decades. The Chirp signal, which has optimum autocorrelation, is a popular choice in radar systems. By encoding communication data into Chirp signals, the scholars have done a lot of research in the field of JRC signal design and have achieved many advances. In literature [9], the down-Chirp linearly frequency modulation and up-Chirp linearly frequency modulation were proposed to realize the binary information. In literature [10-12], the Chirp-BPSK signal was proposed as a compromise solution between communication and detection, but the detection performance was interfered with information data. A novel Chirp-MSK modulation was adopted to construct JRC signal, although the communication performance was perfect, the detection resolution of the JRC signal was worsen compared with the classical Chirp radar waveform [13-17]. Some algorithms were proposed for increasing the communication throughput of the Chirp-MSK signal [15]. Literature [18, 19] proposed the orthogonal frequency division OFDM- 


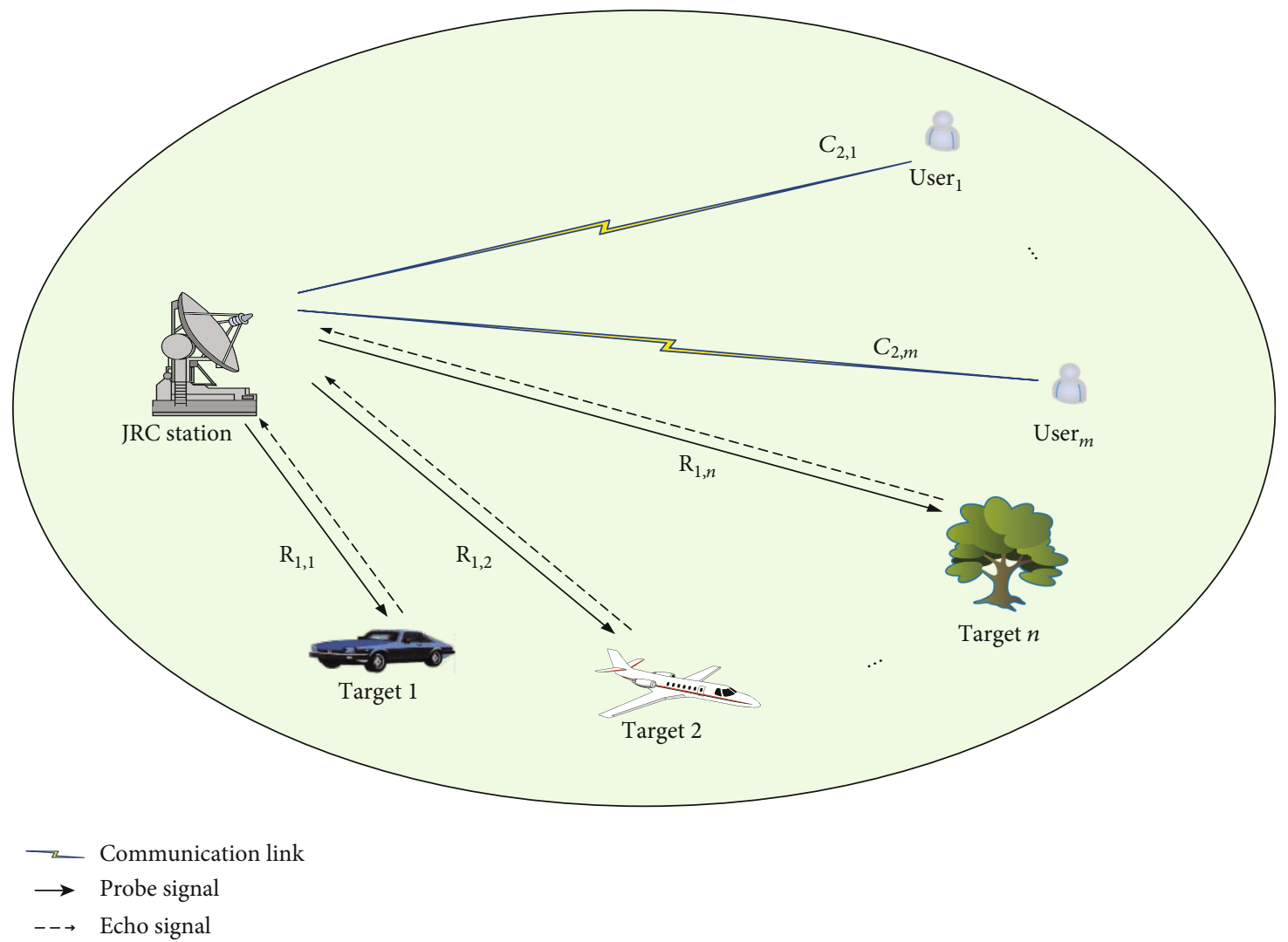

FIgURE 1: The diagram of JRC.

Chirp signal, which has a better peak-to-average-power ratio level and larger time-bandwidth product, and the closedform expression of AF was derived to evaluate the designed waveforms. In literature [20], the author derived the AFs of hyperbolic Chirp signal and Chirp signal, and the hyperbolic Chirp signal has superior Doppler tolerance in the JRC scenario. In literature [21-23], a JRC waveform based on continuous phase modulation (CPM) and Chirp was proposed, the AF of the integrated signal was derived, and the trade-off between radar performance and communication performance via adjustable parameters were analysed. In literature [24], the integrated waveform was generated by CPM and poly-phase coded frequencymodulated radar; the $\mathrm{AF}$ and power spectrum were studied. In literature [25], the approximate orthogonal Chirp signal was guaranteed based on the positive and negative modulation frequency, the JRC signal was realized by combining the $\pi / 4$-QPSK modulation, and the information rate was limited and corresponded to the Chirp rate. As an extension, under various constraints, optimal power allocation schemes were proposed for JRC systems in [26-28], and different waveform design criteria were developed to reduce the total power consumption of the JRC system significantly.

The prior contributions have greatly promoted the technological progress of JRC, but there are still problems such as the performance degradation of $\mathrm{AF}$, deterioration of range resolution and Doppler resolution, inflexible parameter modulation, and limited communication rate. The ChirpBPSK signal is designed for the JRC method in literature $[11,12,29]$, although it has the advantages in transmitting information, but the phase shift mutation of Chirp-BPSK arouses performance degradation of radar.

In this paper, we propose a new JRC signal by modulating improved Binary Phase Shift Keying (BPSK) data into Chirp signal and utilizing hybrid chaotic spread spectrum code (CSSC). By doing so, the JRC signal has excellent rangeDoppler resolution and a high communication rate. Our contributions are summarized as follows:

(1) We derive the mathematic equations of the Chirp signal, Chirp-BPSK signal, and our JRC signal. The JRC signal has excellent radar detection performance and communication performance

(2) We derive the spectrums of the Chirp-BPSK signal and JRC signal and analyse the accumulations of phase shift. It shows that the JRC signal has less distortion and extension in theory

(3) We derive the short-time Fourier transform (STFT) of Chirp-BPSK signal and JRC signal, and the AF characters of Chirp-BPSK signal and JRC signal are analysed by time-frequency distribution methods. The JRC signal's time-frequency distribution is analysed, and it reveals that the JRC signal has more excellent radar resolution 
(4) Different from the prior contributions, the communication rate of the JRC signal is enhanced significantly without deterioration of radar performance

The rest of the paper is organized as follows. Section 2 reviews the traditional Chirp and Chirp-BPSK expressions, presents our novel JRC model, and derives mathematical expressions. In Section 3 we compare the hybrid CSSC with other types of spreading codes in autocorrelation performance. In Section 4, we derive the spectrum equations of the Chirp signal, Chirp-BPSK signal, and JRC signal and analyse the communication and radar performances of the above signals. Section 5 provides computer simulation and verifies our discussion in Section 4. The conclusion is drawn in Section 6.

1.1. Notations. The JRC signal and Chirp-BPSK signal are designed on the basis of the Chirp signal. The Chirp signal, Chirp-BPSK signal, and JRC signal are denoted by $s_{\text {Chirp }}(t)$, $s_{\text {Chirp-BPSK }}(t)$, and $s_{\text {JRC }}(t)$. The Fourier transform of $s_{\text {Chirp }}(t)$, $s_{\text {Chirp-BPSK }}(t)$, and $s_{\text {JRC }}(t)$ is $S_{\text {Chirp }}(f), S_{\text {Chirp-BPSK }}(f)$, and $S_{\text {JRC }}(f)$. For the Chirp signal, the symbol $B$ is the bandwidth, $T$ is the duration width, and $f$ is the frequency.

\section{System Model}

Since the Chirp signal has a low Doppler sensitivity, it is a popular choice in radar systems. And the paper considers the Chirp signal as the baseline for our JRC waveform. We review the equations of Chirp signal and Chirp-BPSK signal, and we derive our JRC signal equation. For the duration width $T$, the Chirp signal linearly sweeps bandwidth $B$, so the equation of Chirp signal is

$s_{\text {Chirp }}(t)=A_{\text {Chirp }} \cdot \exp \left(j \cdot\left(2 \pi \cdot f_{o} \cdot t+j \cdot \pi \cdot u \cdot t^{2}+\Omega_{0}\right)\right), \quad 0 \leq t \leq T$,

where $A_{\text {Chirp }}$ is the signal amplitude, $\Omega_{0}$ is the original phase of the signal, $f_{0}$ is the initial frequency, and $u$ is the chirp rate, while $|u|=B / T$. In order to simplify the discussion, the paper set amplitude $A_{\text {Chirp }}=1, \Omega_{0}=0$, and $f_{0}=0$. For the Chirp signal, the instantaneous frequency $f(t)=u \cdot t$. In this paper, we suppose that the Chirp rate $u$ is positive, and the instantaneous frequency increases with time. The equation of Chirp signal is rewritten as:

$$
s_{\text {Chirp }}(t)=\exp \left(j \cdot \pi \cdot u \cdot t^{2}\right), \quad 0 \leq t \leq T,
$$

where the signal spectrum is distributed between 0 and $B$.

2.1. Chirp-BPSK Signal. The chirp signal is also a common scheme to transmit binary data, which is named chirp modulation. The binary data is transmitted by mapping the bits into positive $u$ and negative $u$, and one Chirp signal can only transmit one bit [9]. Similarly, in order to increase the communication rate, multiple binary data are multiplying with one Chirp signal, and the Chirp-BPSK signal can be built by using BPSK bit mapping to embed communication data into the Chirp signal [10-12]. The Chirp-BPSK signal is expressed as:

$$
\begin{aligned}
s_{\text {Chirp-BPSK }}(t) & =b(t) \cdot s_{\text {Chirp }}(t)=\sum_{i=1}^{N} b_{i} \cdot u\left(t-i T_{b}\right) \cdot s_{\text {Chirp }}(t) \\
& =\sum_{i=1}^{N} b_{i} \cdot u\left(t-i T_{b}\right) \cdot \exp \left(j \cdot \pi \cdot u \cdot t^{2}\right),
\end{aligned}
$$

where $b(t)$ denotes $N$ bit BPSK modulation data, as well as $b_{i}= \pm 1$ is the $i$-th bit of the BPSK data, $T_{b}$ stands for pulse width of BPSK bit, and $u(t)=\left\{\begin{array}{ll}1, & 0<t<T_{b} \\ 0, & \text { elsewhere }\end{array}\right.$ is a rectangular pulse.

The pulse width $T$ of the Chirp signal can be expressed as $T=N \cdot T_{b}$, so a Chirp-BPSK signal can transmit $N$ bits of information data. The value of the $i$-th information data is +1 or -1 , it means adding a phase shift $\theta_{i}$ to the Chirp signal. According to the different BPSK bit, $\theta_{i}$ varies between $\pi / 2$ and $-\pi / 2$. The Chirp-BPSK can be rewritten as follows [11]:

$$
s_{\text {Chirp-BPSK }}(t)=\sum_{i=1}^{N} u\left(t-i T_{b}\right) \cdot \exp \left(j \cdot \pi \cdot u \cdot t^{2}+j \cdot \theta_{i}\right) \text {. }
$$

The Chirp-BPSK signal is designed based on the Chirp signal, and it has excellent communication performance. But from the radar's viewpoint, the radar detection performance deteriorates significantly due to communication interference.

2.2. JRC Waveform. Different from standard BPSK modulation whose phase offset is $\pi$, we suppose the phase offset of binary BPSK data decreases to $\theta_{k}\left(2 \theta_{k}<\pi\right)$, and the phase varies between $\theta_{k}$ or $-\theta_{k}$, respectively, an improved BPSK modulation is formed [26]. When the phase offset $2 \theta_{k}$ is 0 , the improved BPSK becomes a constant signal. When the phase offset $2 \theta_{k}$ is $\pi / 2$, the improved BPSK signal becomes orthogonal signal. When the phase offset $2 \theta_{k}$ is $\pi$, the improved BPSK becomes a conventional BPSK signal. Thus, constant signal, orthogonal signal, and BPSK signal can be considered as special cases of improved BPSK modulation. The improved BPSK modulation has a smaller phase offset, so the distance of the binary constellation is smaller. In additive white Gaussian noise (AWGN) channel, the power spectral density is $N_{0} / 2$, the Bit Error Ratio (BER) of BPSK is

$$
P_{\mathrm{BPSK}}=Q\left(\sqrt{\frac{2 \varepsilon_{b}}{N_{0}}}\right),
$$

where $\varepsilon_{b}$ is unit bit energy. 


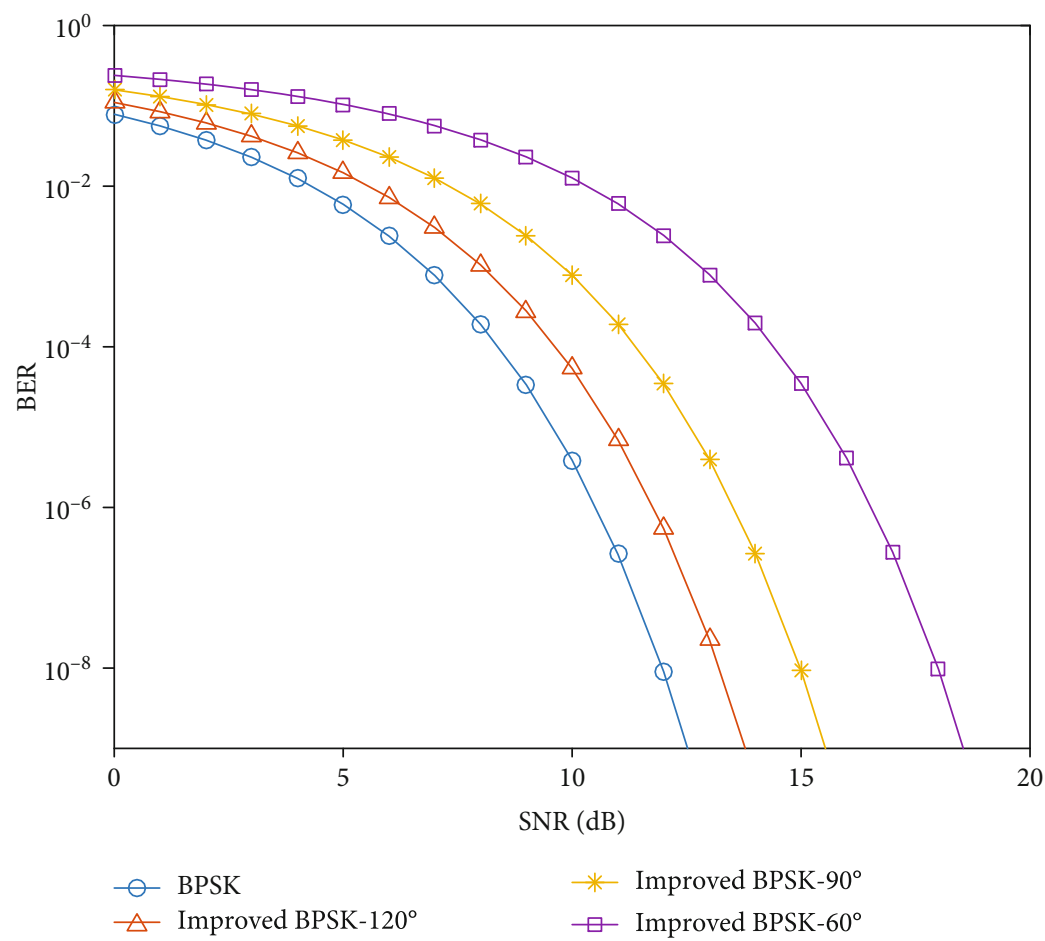

FIGURE 2: The BER of improved BPSK versus different angles.

Similarly, in the AWGN channel, the BER of the improved BPSK is given by [29]:

$$
P_{\text {improved-BPSK }}=Q\left(\sqrt{\frac{2 \varepsilon_{b} \sin ^{2}\left(\theta_{k}\right)}{N_{0}}}\right)
$$

Comparing between $P_{\mathrm{BPSK}}$ and $P_{\text {improved-BPSK}}$, we can deduce the conclusion that item $\sin ^{2}\left(\theta_{k}\right)$ decreases the BER performance, and the BER performance of improved BPSK is worse. In the AWGN channel, the BER of binary improved BPSK modulation signals with different $2 \theta_{k}$ are shown in Figure 2. The BER of improved BPSK versus different SNR.

With the decrease of the $2 \theta_{k}$, the BER of the improved BPSK deteriorates. The improved BPSK- $60^{\circ}\left(2 \theta_{k}\right.$ is $\left.\pi / 3\right)$ has a performance loss of $6 \mathrm{~dB}$ compared to the traditional BPSK $\left(2 \theta_{k}\right.$ is $\left.\pi\right)$. When the radio propagation SNR is very high, the BER loss of improved BPSK is effectively compensated. Hence, it is easy to derive the improved ChirpBPSK signal:

$$
\begin{aligned}
s_{\text {improved-Chirp-BPSK }}(t) & =\sum_{i=1}^{N} b_{i} \cdot u\left(t-i T_{b}\right) \cdot s_{\text {Chirp }}(t) \cdot \exp \left(j \cdot \theta_{k}\right) \\
& =\sum_{i=1}^{N} b_{i} \cdot u\left(t-i T_{b}\right) \cdot \exp \left(j \cdot \pi \cdot u \cdot t^{2}+j \cdot \theta_{k}\right) \\
& =\sum_{i=1}^{N} u\left(t-i T_{b}\right) \cdot \exp \left(j \cdot \pi \cdot u \cdot t^{2}+j \cdot b_{i} \cdot \theta_{k}\right),
\end{aligned}
$$

where the phase offset $b_{i} \cdot \theta_{k}$ is introduced by the $i$-th communication data. If $b_{i}=1$, the phase offset is $\theta_{k}$, while $b_{i}=-1$, phase offset is $-\theta_{k}$.

The performance loss of improved BPSK must be compensated effectively, so we apply the spread spectrum code to compensate for the JRC waveform. Spread spectrum code is widely used in communication, radar, navigation, and so on. Standard spread spectrum codes include (1) $m$ sequence, (2) Gold sequence, and (3) CSSC. The spread gain of the spread spectrum code can compensate for the loss of improved BPSK propagation. When the spread spectrum factor is $M$, and the spread spectrum gain is $10 \log M$, the appropriate $M$ value will be sufficient to compensate for the performance loss caused by the improved BPSK. In our JRC signal, the performance loss caused by improved BPSK can be compensated by high Chirp radar SNR and CSSC.

After the spread spectrum processing of the improved Chirp-BPSK signal, the expression of the JRC signal can be obtained as follows:

$$
\begin{aligned}
s_{\mathrm{JRC}}(t) & =\sum_{i=1}^{N} \sum_{k=1}^{M} u_{c}\left(t-i T_{b}-k T_{c}\right) \cdot s_{\mathrm{Chirp}}(t) \cdot \exp \left(j \cdot b_{i} \cdot c_{k} \cdot \theta_{k}\right) \\
& =\sum_{i=1}^{N} \sum_{k=1}^{M} u_{c}\left(t-i T_{b}-k T_{c}\right) \cdot \exp \left(j \cdot\left(\pi \cdot u \cdot t^{2}+b_{i} \cdot c_{k} \cdot \theta_{k}\right)\right),
\end{aligned}
$$

where $T_{c}$ is the duration of the unit spread spectrum Chip, $u_{c}(t)$ is also a rectangular pulse $u_{c}(t)=\left\{\begin{array}{ll}1, & 0<t<T_{c} \\ 0, & \text { elsewhere }\end{array}\right.$, $c_{k}= \pm 1$ is the chip of the spread spectrum sequence, and 
the value $b_{i}$ of the data is -1 or +1 . The value of $b_{i} c_{k}$ range is -1 or +1 . The phase shift of the JRC waveform is not $\pi / 2$ or $-\pi / 2$, but it is $\theta_{k}$ or $-\theta_{k}$.

\section{Spread Spectrum Code}

In this section, we briefly describe the main characteristics of the $m$ sequence, Gold sequence, and CSSC. Compared with $m$ sequence and Gold sequence, CSSC has perfect correlation function, flexible length, and superior confidentiality [30]. Considering the perfect performance of CSSC, we adopt CSSC as a spread spectrum sequence for our JRC signal.

When CSSC is used as spread spectrum codes, discrete chaotic systems are usually used to generate discrete spread spectrum sequences of arbitrary length. CSSC include Logistic sequence, improved Logistic sequence, Tent sequence, and Chebyshev sequence [31]. The length of CSSC is no longer limited to $2^{n}-1$ like $m$ sequence and Gold sequence, and extended number of nonrepeating spread sequences can be generated by fine-tuning the initial value. In order to improve the complexity and randomness of CSSC, improved Logistic chaotic sequences and Chebyshev chaotic sequences are adopted to generate hybrid CSSC [30]. The definition of improved Logistic chaotic sequence is as follows:

$$
x_{n+1}=1-k \cdot x_{n}^{2},-1<x_{n+1}<1,
$$

where $x_{n}$ is the current state of the sequence, $x_{n+1}$ is the next state of the sequence, $k=2$ is the fractal parameter, and $x_{1}=0.7$ is the initial value.

Chebyshev chaotic sequence is defined as follows:

$$
y_{n+1}=\cos \left(\omega \operatorname{arcos} y_{n}\right),-1 \leq y_{n} \leq 1
$$

where $y_{n}$ is the current state of the sequence, $y_{n+1}$ is the next state of the sequence, $\omega=8$ is the order number, and $y_{1}=0.5$ is the initial value.

The above iterative sequences are a nonperiodic, and they are in a chaotic state. Hybrid CSSC can be obtained by xor processing of improved Logistic chaotic sequence and Chebyshev chaotic sequence, and the hybrid CSSC equation is $z_{n}=x_{n} \oplus y_{n}$. Hybrid CSSC is of great value in communication and radar detection.

In the paper, the 127-bits spread spectrum sequences are employed in the JRC signal. Auto-correlation and crosscorrelation functions of $m$ sequence, Gold sequence, and hybrid CSSC sequences are compared, and the results are shown in Figure 3.

Figure 3 proves that the hybrid CSSC has better crosscorrelation and auto-correlation performance. Hybrid CSSC have significantly more numerous than $m$ sequences and Gold sequences. Through the above analysis, the hybrid CSSC has the advantages in flexible length, huge numbers, and high confidentiality, and these characters are beneficial to the security of the JRC signal in the paper.

\section{Performance Analysis of JRC Signal}

The performance analysis of the JRC signal includes communication performance and radar performance. The communication performance emphasizes data transmission efficiency, while the radar performance focuses on the accuracy of measurement. In this paper, the measurement of communication performance is BER and spectrum, and the measurement of radar performance is AF of signal.

4.1. Communication Performance. It is known that, in the AWGN channel, the JRC signal combined with Chirp signal and digital modulation, has the BER equivalent to the original digital modulation [32]. The BER results are shown in Figure 2, the Chirp-BPSK and BPSK modulations have the equal output under the same SNR condition. Furthermore, in the same channel condition, our JRC signal has a larger tolerance for interference resistance, and the BER results can be compensated by hybrid CSSC in our strategy.

Next, we derive the spectrum functions for the Chirp signal, Chirp-BPSK signal, and our JRC signal. The communication performance can be analysed according to the spectrum. The expression of Chirp's spectrum can be calculated by [33]:

$S_{\text {Chirp }}(f)=\frac{\exp \left(-j \cdot \pi \cdot f^{2} / u\right)\left[\left(C\left(x_{2}\right)-C\left(x_{1}\right)\right)+j \cdot\left(S\left(x_{2}\right)-S\left(x_{1}\right)\right)\right]}{\sqrt{2 \cdot u}}$,

where the $C(x)$ and $S(x)$ stand for Fresnel functions $C(x)=$ $\int_{0}^{x} \cos \left(\pi \cdot v^{2} / 2\right) d v \quad$ and $S(x)=\int_{0}^{x} \sin \left(\pi \cdot v^{2} / 2\right) d v, \quad x_{2}=$ $\sqrt{(B \cdot T / 2)}(1+(f / B / 2))$, and $x_{1}=\sqrt{(B \cdot T / 2)}(1-(f / B / 2))$.

We suppose one Chirp-BPSK signal carrying $N$ bit communication data, then we get the expression of Chirp-BPSK's spectrum (Appendix A):

$S_{\text {Chirp-B }}(f)=\frac{\sum_{i=1}^{N} \exp \left(j \cdot \theta_{i}-j \cdot \pi \cdot f^{2} / u\right)\left[\left(C\left(x_{4}\right)-C\left(x_{3}\right)\right)+j \cdot\left(S\left(x_{4}\right)-S\left(x_{3}\right)\right)\right]}{\sqrt{2 \cdot u}}$,

where $x_{3}=\sqrt{2 \cdot u}\left((i-1) T_{b}-f / u\right), x_{4}=\sqrt{2 \cdot u}\left(i \cdot T_{b}-f / u\right)$, and $\theta_{i}= \pm \pi / 2$.

We suppose the JRC signal carrying $N$ bit data, spread spectrum factor of hybrid CSSC is $M$, which means the JRC signal can bring $M \times N$ microbits, the phase $\theta_{k}= \pm \theta(\theta<\pi / 2)$, and the expression of JRC's spectrum can be written as:

$S_{\mathrm{JRC}}(f)=\frac{\sum_{k=1}^{N M} \exp \left(j \cdot \theta_{k}-j \cdot \pi \cdot f^{2} / u\right)\left[\left(C\left(x_{6}\right)-C\left(x_{5}\right)\right)+j \cdot\left(S\left(x_{6}\right)-S\left(x_{5}\right)\right)\right]}{\sqrt{2 \cdot u}}$,

where $x_{5}=\sqrt{2 \cdot u}\left((i-1) T_{c}-f / u\right), x_{6}=\sqrt{2 \cdot u}\left(i \cdot T_{c}-f / u\right)$, and $\theta_{k}= \pm \theta$.

We carry out a comparison of (11) and (11) and (13), the three spectrum functions are compounded of Fresnel function and other function modulation. The spectrum functions of the Chirp-BPSK signal and the JRC signal 


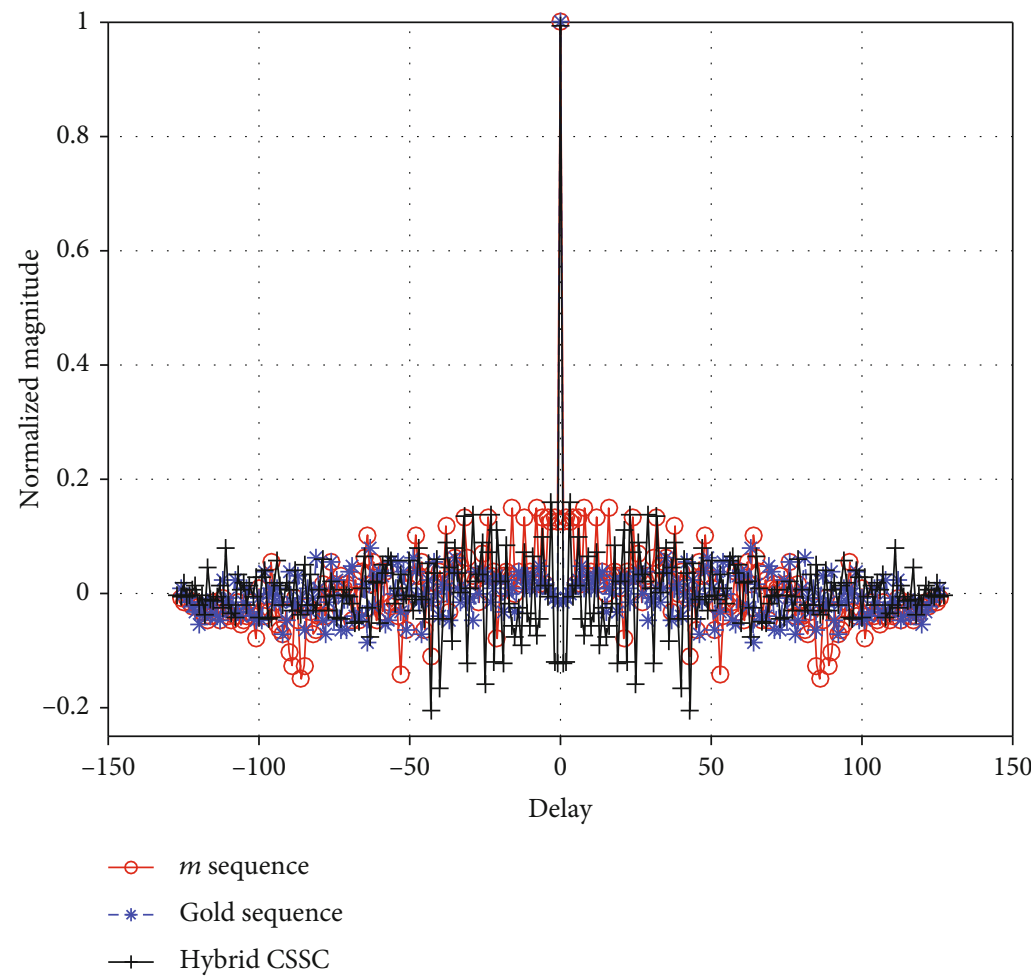

(a)

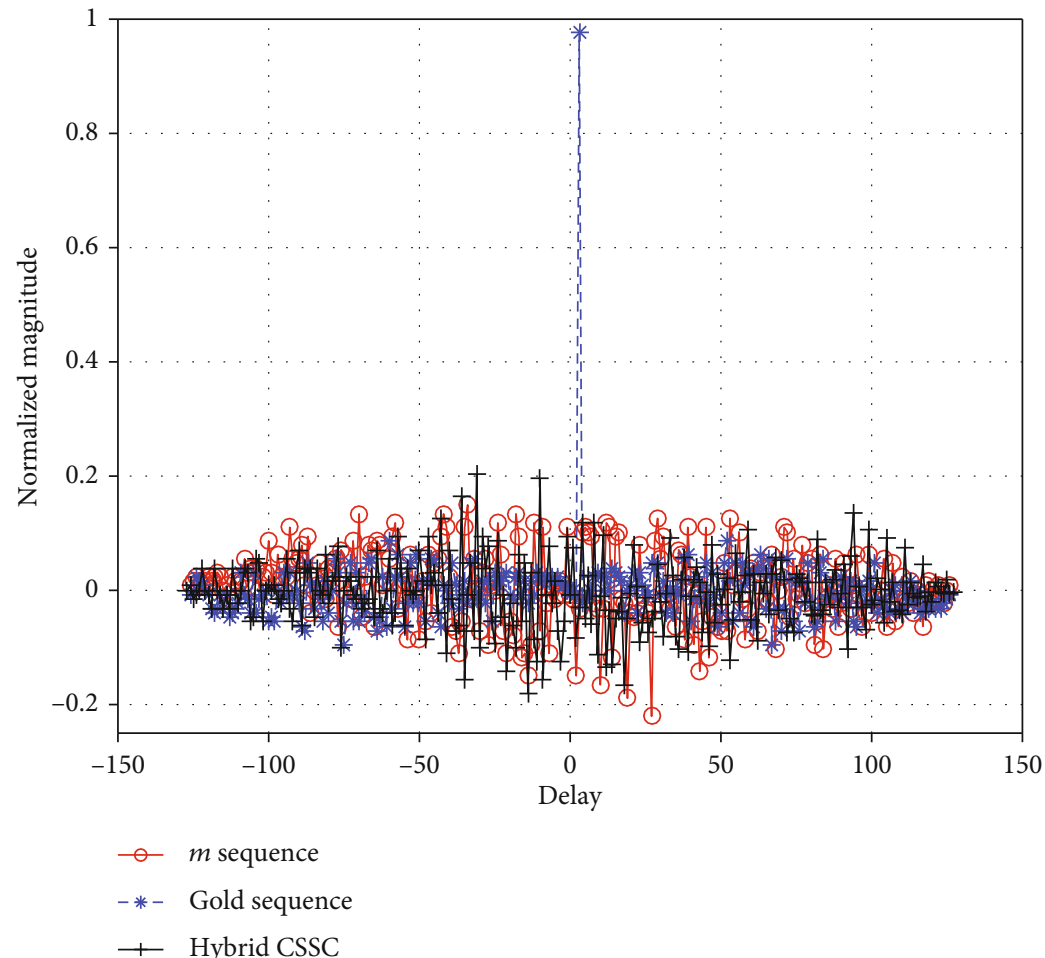

(b)

FIGURE 3: Comparison of aperiodic correlation functions of 127-bit spread spectrum sequences: (a) auto-correlation functions; (b) crosscorrelation functions. 
are the superposition of multiple element signal spectrum, the greater phase shift introduces more serious spectrum distortion and extension. The phase shift $\theta_{k}$ of the JRC signal is lesser than the phase shift $\theta_{i}$ of the ChirpBPSK signal, so the JRC signal's spectrum function should have lesser distortion and extension than the Chirp-BPSK signal's spectrum function theoretically. Supposed the phase shift $\theta_{k}$ of the JRC signal gradually decreases to null, and its spectrum gradually tends to the Chirp signal spectrum. So the JRC signal has better communication performance.

4.2. Radar Performance. The AF is an important element in the radar performance field, which is used to estimate the range accuracy and Doppler accuracy. In this paper, we appraise the radar performance by employing the AF performance. The AF is defined as [10]:

$$
\chi\left(\tau, f_{d}\right)=\left|\int_{0}^{T} s(t) \cdot s^{*}(t-\tau) \cdot e^{j \cdot 2 \pi \cdot f_{d} \cdot t} d t\right|
$$

where $\tau$ and $f_{d}$ are the time delay and Doppler shift of radar echo.

The AF of the ideal radar signal appears as a "pushpin" shape, which has an infinite impact value only at point ( $\left.\tau=0, f_{d}=0\right)$, null others. In theory, as long as there is no time delay or Doppler shift, the radar can provide an accurate target detection. In practice, the ideal AF is impossible, and the AF of the Chirp signal is standard oblique blade shape. The JRC signal is carrying the communication data, which inevitably has the distortion of the AF. The AF performance of the three signals are analysed as follows.

The AF of classical Chirp signal is [33]

$$
\begin{aligned}
\chi_{\text {Chirp }}\left(\tau, f_{d}\right) & =\left|\int_{0}^{T} \exp \left(j \cdot \pi \cdot u \cdot t^{2}\right) \cdot \exp \left(-j \cdot \pi \cdot u \cdot(t-\tau)^{2}\right) \cdot e^{j \cdot 2 \pi \cdot f_{d} \cdot t} d t\right| \\
& =\left|\left(1-\frac{|\tau|}{T}\right) \cdot \frac{\sin \left[\pi \cdot\left(u \cdot \tau+f_{d}\right) \cdot(1-|\tau| / T)\right]}{\pi \cdot\left(u \cdot \tau+f_{d}\right) \cdot(1-|\tau| / T)}\right|
\end{aligned}
$$

We can derive the AF of Chirp-BPSK from (14):

$$
\begin{aligned}
\chi_{\text {Chirp-BPSK }}\left(\tau, f_{d}\right)= & \mid \int_{0}^{T} \sum_{i=1}^{N} \sum_{i=1}^{N} u\left(t-i T_{b}\right) \\
& \cdot \exp \left(j \cdot \pi \cdot u \cdot t^{2}+j \cdot \theta_{i}\right) \cdot u\left(t-\tau-i T_{b}\right) \\
& \cdot \exp \left(-j \cdot \pi \cdot u \cdot(t-\tau)^{2}-j \cdot \theta_{i}\right) \cdot e^{j \cdot 2 \pi \cdot f_{d} \cdot t} d t \mid,
\end{aligned}
$$

where $\theta_{i}= \pm \pi / 2$.
Similarly, the AF of the JRC signal is calculated as:

$$
\begin{aligned}
\chi_{\mathrm{JRC}}\left(\tau, f_{d}\right)= & \mid \int_{0}^{T} \sum_{k=1}^{N M} \sum_{k=1}^{N M} u\left(t-k T_{c}\right) \cdot \exp \left(j \cdot \pi \cdot u \cdot t^{2}+j \cdot \theta_{k}\right) \\
& \cdot u\left(t-\tau-k T_{c}\right) \cdot \exp \left(-j \cdot \pi \cdot u \cdot(t-\tau)^{2}-j \cdot \theta_{k}\right) \\
& \cdot e^{j \cdot 2 \pi \cdot f_{d} \cdot t} d t \mid,
\end{aligned}
$$

where $\theta_{k}= \pm \theta$.

We present comparisons of (15) and (16) and (17), the AF of Chirp signal is simple and clear, while the AFs of Chirp-BPSK and JRC are extremely complicated because of communication data. Therefore, the time-frequency analysis is employed to explain the AF characteristics. The short-time Fourier transform (STFT) is the main method of linear timefrequency analysis $[13,14]$. The basic idea of STFT is to use a fixed sliding time window to cut the original signal into many tiny time intervals. The signals in the truncated window are nearly stationary, and the Fourier transform of each signal is performed to obtain the signal spectrum of each time period. The STFT for a given signal $s(t)$ is defined as:

$$
\operatorname{STFT}_{s}(t, f)=\int s(\tau) \cdot w^{*}(\tau-t) \cdot \exp (-j \cdot 2 \pi \cdot f \cdot t) d \tau,
$$

where $w(t)$ is generally a real window function, namely, $w^{*}$ $(t)=w(t)$. In the paper, we suppose the time-window of STFT is a Gaussian window, in which the number of STFT is 1024 , and the window overlap is 1000 . We define that the $S_{p}(t, f)$ is the spectrogram for signal $s(t)$ :

$$
S_{p}(t, f)=\left|\operatorname{STFT}_{s}(t, f)\right|^{2} .
$$

The STFT and $S_{p}(t, f)$ for Chirp-BPSK are expressed as (Appendix B):

$$
\begin{aligned}
\operatorname{STFT}_{\text {Chirp-BPSK }}(t, f)= & \frac{2 \cdot \sigma \cdot \sqrt{\pi}}{\sqrt{\left(2 \cdot \pi \cdot \sigma^{2} \cdot \mu\right)^{2}+1}} \int s_{\text {Chirp-BPSK }}(\tau) \\
& \cdot w^{*}(\tau-t) \cdot \exp (-j \cdot 2 \pi \cdot f \cdot t) d \tau,
\end{aligned}
$$

$S_{p}(t, f)=A^{2} \exp \left\{\frac{2 \cdot\left[\pi \cdot \sigma\left(f-\left(u \cdot t+\sum_{i=1}^{N} u\left(t-i T_{b}\right) \cdot b_{i} / 4 T_{b}\right)\right]^{2}\right.}{\left(2 \cdot \pi \cdot \sigma^{2} \cdot \mu\right)^{2}+1}\right\}$.

The time-frequency function of Chirp -BPSK is

$$
f=u \cdot t+\sum_{i=1}^{N} u\left(t-i T_{b}\right) \cdot \frac{b_{i}}{4 T_{b}} .
$$

If the $i$-th symbol is -1 , the phase shift is $\pi / 2$ during the duration of the symbol; while the $i$-th symbol is 1 , the 
corresponding phase shift is $-\pi / 2$. Spectral energy is mainly distributed between lines $f=u \cdot t-\left(1 / 4 T_{b}\right)$ and $f=u \cdot t+$ $\left(1 / 4 T_{b}\right)$. When the symbol varies between -1 and +1 , the signal is affected by the shift cumulative term $\sum_{i=1}^{N} u\left(t-i T_{b}\right)$ - $\left(b_{i} / 4 T_{b}\right)$, which leads to the diffusion of the AF. Consequently, the radar performance of range resolution and velocity resolution decreases. With the communication rate of the Chirp-BPSK signal increasing, the more timefrequency diffusions occur.

According to (21) and (22), while the $b_{i}=0$, the $S_{p}(t, f)$ and time-frequency equation of Chirp signal are derived as:

$$
\begin{gathered}
S_{p}(t, f)=A^{2} \cdot \exp \left\{\frac{2 \cdot[\pi \cdot \sigma(f-u \cdot t)]^{2}}{\left(2 \cdot \pi \cdot \sigma^{2} \cdot \mu\right)^{2}+1}\right\}, \\
f=u \cdot t .
\end{gathered}
$$

For the Chirp signal, the spectral energy is linearly distributed, which leads to the linear distribution of the AF. According to (21) and (22), the $S_{p}(t, f)$ and time-frequency equation of our JRC signal are

$$
S_{p}(t, f)=A^{2} \exp \left\{\frac{2 \cdot\left[\pi \cdot \sigma\left(f-\left(u \cdot t+\sum_{k=1}^{N M} u_{c}\left(t-k T_{c}\right) \cdot b_{k} / 2 \cdot L \cdot T_{c}\right)\right]^{2}\right.}{\left(2 \cdot \pi \cdot \sigma^{2} \cdot \mu\right)^{2}+1}\right\}
$$

$$
f=u \cdot t+\sum_{k=1}^{N M} u_{c}\left(t-k T_{c}\right) \cdot \frac{b_{k}}{2 \cdot L \cdot T_{c}}
$$

If the $k$-th symbol is -1 , the phase shift is $-\pi / L$ during the duration of the symbol; while the $k$-th symbol is 1 , the corresponding phase shift is $\pi / L$. The time-frequency formula of the JRC signal has a frequency shift term $\sum_{k=1}^{N M} u_{c}\left(t-k T_{c}\right)$ $\cdot\left(b_{k} / 2 \cdot L \cdot T_{c}\right)$. However, when $L$ is greater than 2 , the accumulation is smaller than the Chirp-BPSK signal. On the other hand, the data after the spread spectrum is a bit with a value of +1 or -1 , which shows the random distribution characteristics of spread spectrum code. The probability of +1 or -1 appears in the sequence, and the sum of sequences approaches null.

The better the random performance of spread spectrum code is, the more approximate its cumulative sum is null. The effect makes the frequency accumulation approach to null, so that the main energy of the JRC signal timefrequency diagram tends to be on the straight line $f=u \cdot t$. Therefore, the AF distribution of the JRC signal is less diffusion than the AF of the Chirp-BPSK signal. At the same time, when the amount of communication data carried by the JRC signal increases, it has no effect on the accumulation of frequency shift in the time-frequency formula, and the AF characteristics of the JRC signal remain unchanged. So the JRC signal has better radar performance.
TABLE 1: Simulation parameters.

\begin{tabular}{lc}
\hline Parameter & Value \\
\hline$T$ & $20 \mathrm{us}$ \\
$B$ & $10 \mathrm{MHz}$ \\
$f_{s}$ & $500 \mathrm{MHz}$ \\
$u$ & $0.5 \times 10^{12}$ \\
$M$ & 127 \\
$N$ & $50,100,200$ \\
$\theta_{i}$ & $\pi / 2$ \\
$\theta_{k}$ & $\pi / 6$ \\
$L$ & 6 \\
$T_{b}$ & $T / N$ \\
$T_{c}$ & $T_{b} / M$ \\
\hline
\end{tabular}

\section{Simulation and Discussions}

In order to illuminate the viewpoints in Section 4, we employ four kinds of signals (Chirp signal, Chirp-BPSK signal, Chirp-MSK signal, and our JRC signal) to our numerical simulation. The Chirp-MSK signal was adopted to construct a joint radar-communication signal for the perfect spectrum characteristics, attributing to the continuous phase of MSK. The spectrum equation, time-frequency analyse, and AF equation of Chirp-MSK signal are beyond the scope of this article, which are discussed in detail in [13-17]. Compared with the Chirp-BPSK signal and Chirp-MSK signal [13], our JRC signal has better performance in radar detection in theory.

The simulation parameters of the above signals are supposed as Table 1 . The $M$ bits of hybrid CSSC designed in Section 3 are used as the spreading codes; the improved BPSK modulation is used instead of BPSK in the JRC signal. The Chirp-BPSK signal, Chirp-MSK signal, and the JRC signal can transmit $N$ communication bits.

5.1. Computational Complexity. According to (8), the computational complexity of our JRC method is mainly contributed by creating $M$ bits of hybrid CSSC and transmitting $N$ communication bits. Reviewing (9) and (10), we need to perform the $x_{n}, y_{n}$, and $z_{n}$ to obtain the hybrid CSSC, so the computational complexity of creating $M$ bits of hybrid CSSC is $\mathrm{O}(3 M)$. Another complexity operation is embedding $N$ communication bits to one Chirp signal, the computational complexity is $\mathrm{O}(N)$. We obtain the computational complexity of our JRC method is $\mathrm{O}(3 M N)$; the whole complexity is related to the length of hybrid CSSC and count of communication bits.

5.2. Spectrum Performance. The spectrum function of the conventional Chirp signal is made up of Fresnel functions; most of the spectrum energy is concentrated in the narrow Chirp signal bandwidth. Modulation of communication data 


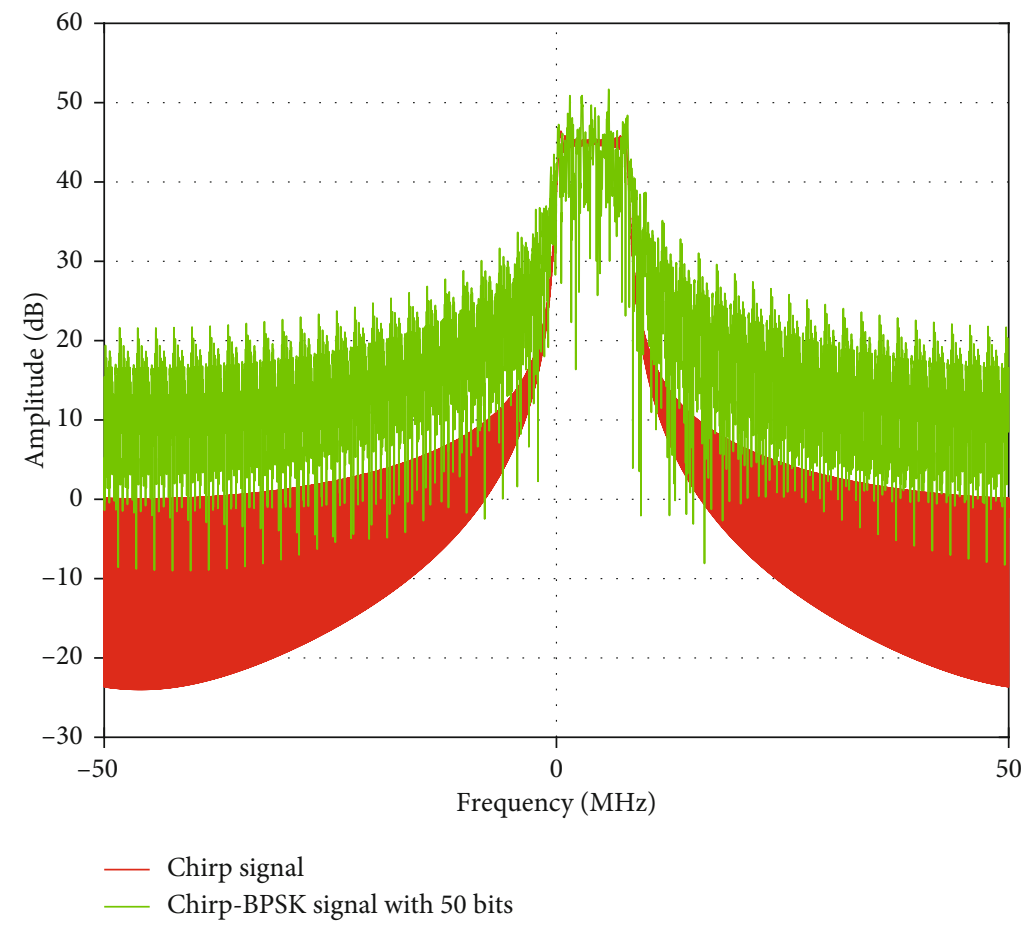

(a)

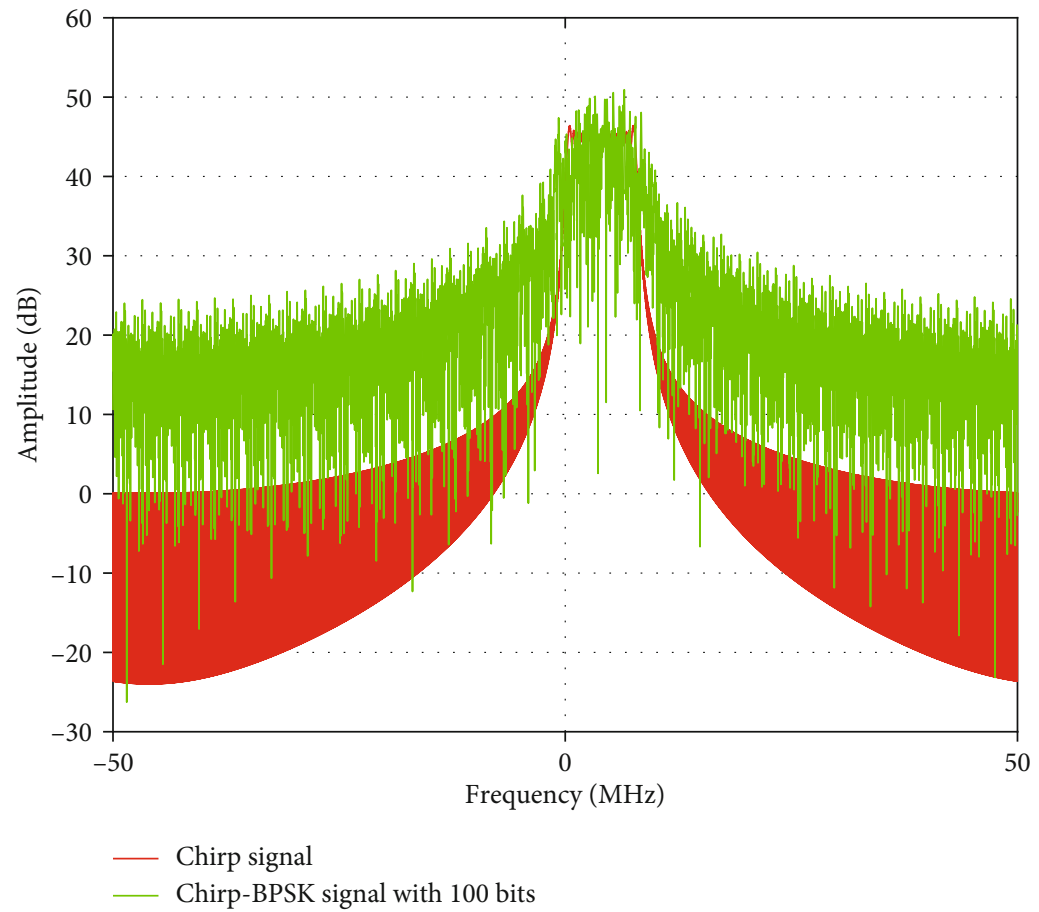

(b)

FIgure 4: Comparison of spectrums for Chirp signal and Chirp-BPSK signal: (a) Chirp and Chirp-BPSK with 50-bit data; (b) Chirp and Chirp-BPSK with 100-bit data.

will lead to energy leakage and spectrum distortion. Figures 4-6 plot the spectrum functions of Chirp signal, Chirp-BPSK signal, Chirp-MSK signal [13], and JRC signal. As shown in Figures 4-6, while carrying the communication bits, the spectrum of Chirp-BPSK signal, Chirp-MSK signal, and JRC signal inevitably have diffusion and fluctuation. The more communication bits, the more spectrum expansion and energy leakage. Compared to Chirp-MSK and JRC, Chirp-BPSK has more spectrum expansion, more energy leakage, larger amplitude fluctuation, and larger 


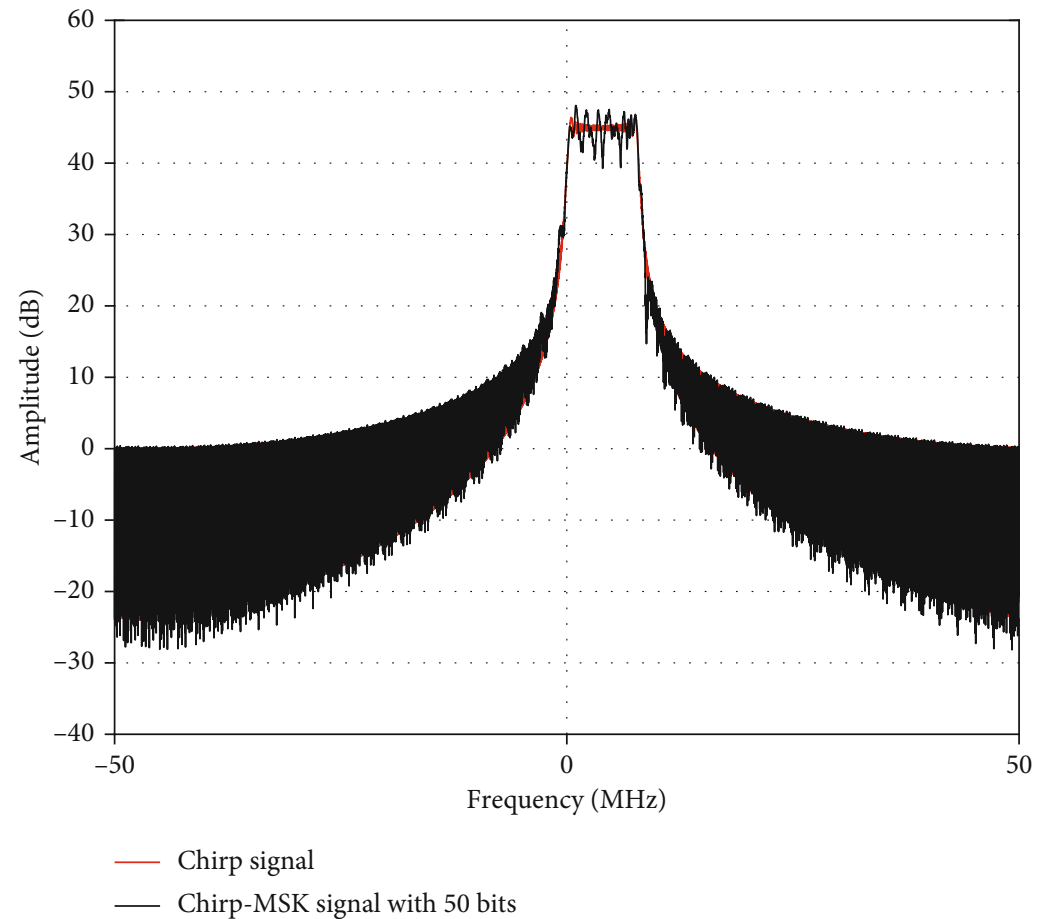

(a)

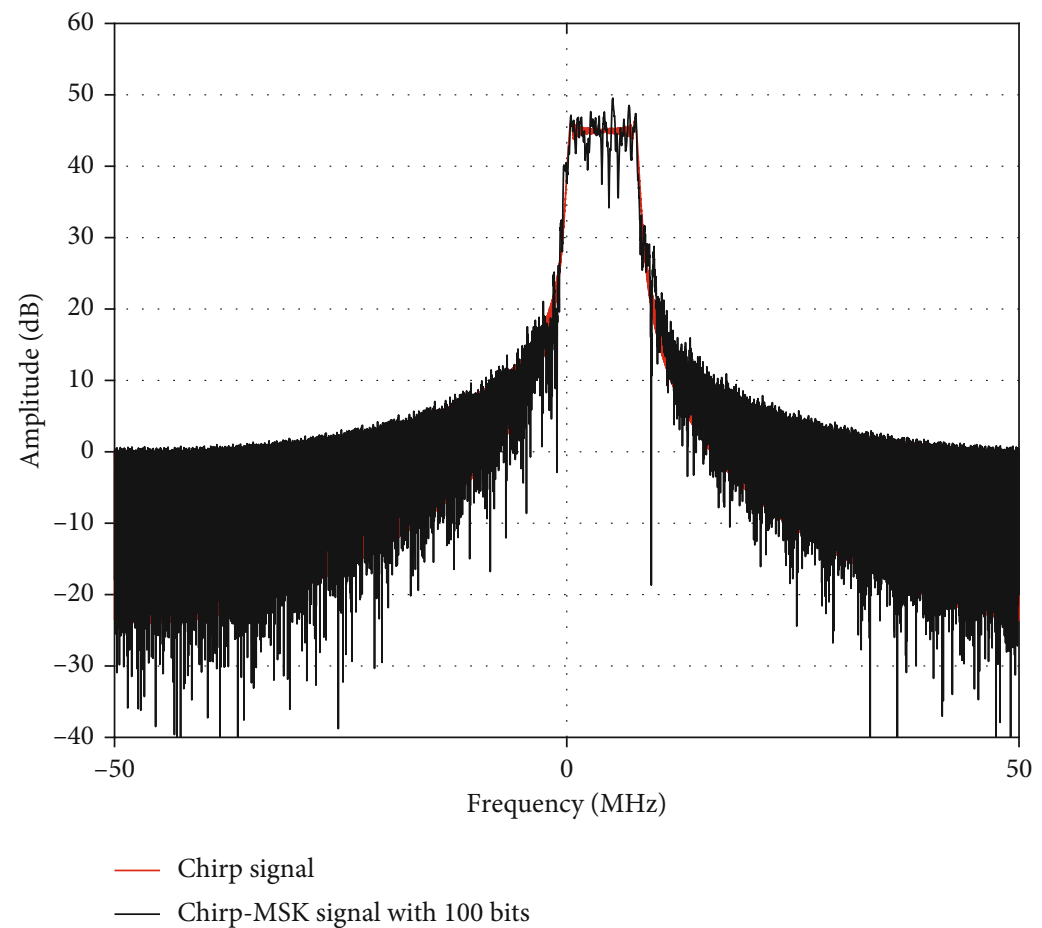

(b)

FIgURE 5: Comparison of spectrums for Chirp signal and Chirp-MSK signal [13]: (a) Chirp and Chirp-MSK with 50-bit data; (b) Chirp and Chirp-MSK with 100-bit data.

interference. As mentioned continuous phase of MSK, the spectrum function of Chirp-MSK has excellent performance in the three signals. And the spectrum leakage and amplitude fluctuation of the JRC signal are improved at the base of Chirp-BPSK. We consider the comparing results; the JRC signal is better than the Chirp-BPSK signal and worse than the Chirp-MSK signal in terms of communication performance. 


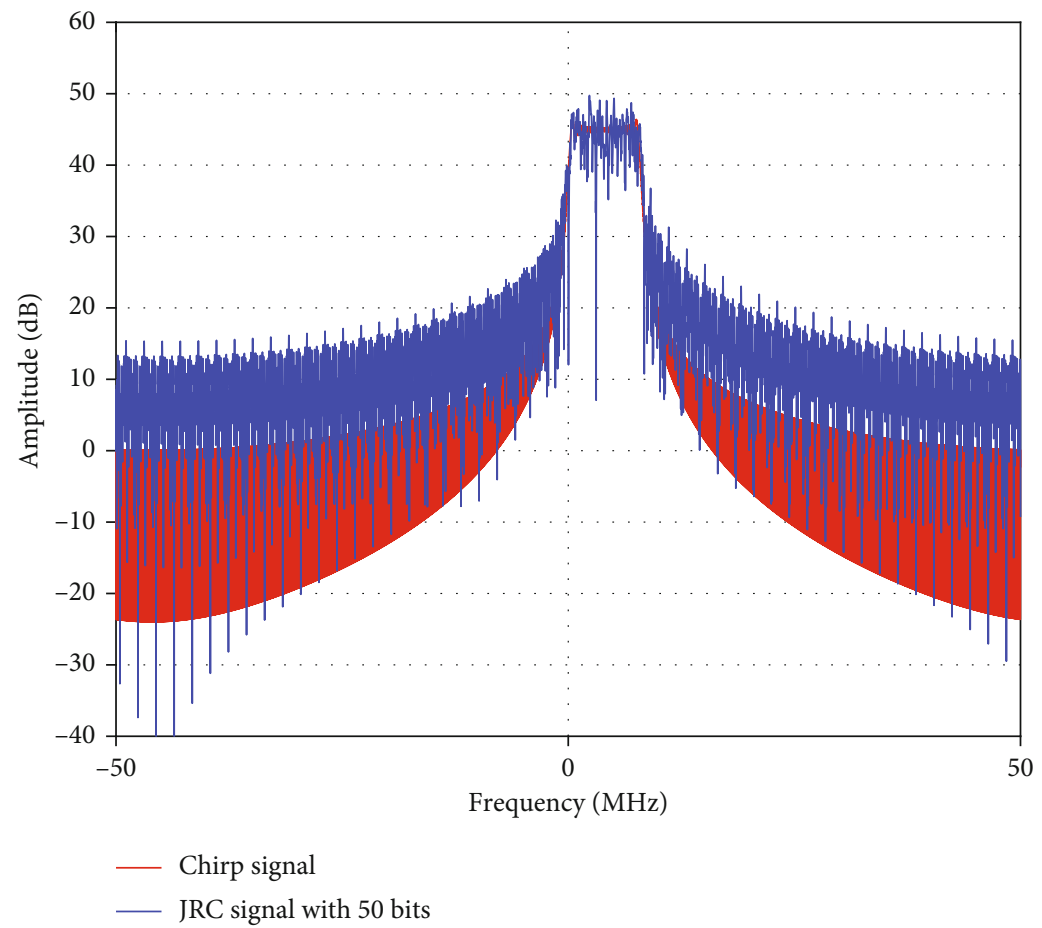

(a)

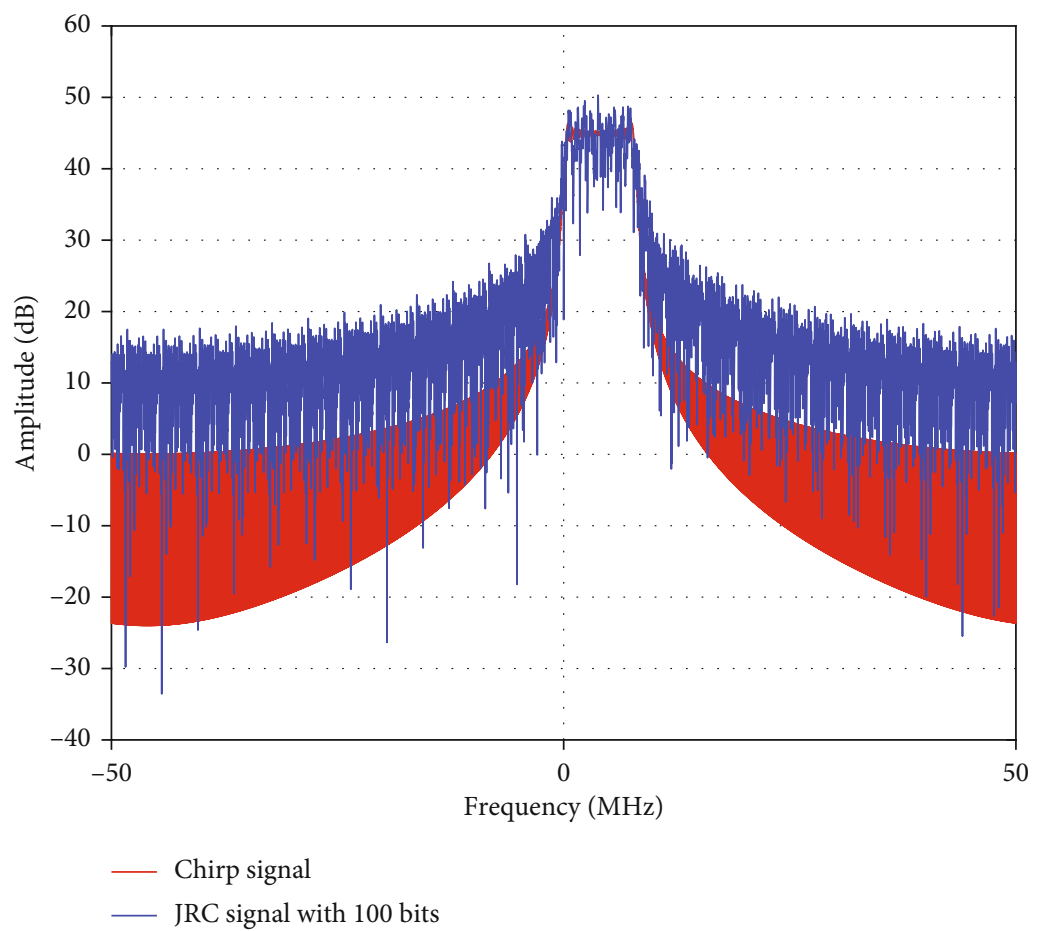

(b)

FIGURE 6: Comparison of spectrums for Chirp signal and JRC signal: (a) Chirp and JRC with 50-bit data; (b) Chirp and JRC with 100-bit data.

5.3. Time-Frequency Performance. Time-frequency analysis graphs for Chirp signal, Chirp-BPSK signal, Chirp-MSK signal, and JRC signal are shown in Figures 7 and 8. As shown in Figure 7, main energy of the Chirp signal and JRC tend to be on the straight line $f=u \cdot t$, while the main energy of the Chirp-BPSK and Chirp-MSK signal is obviously diffused, which lead to the different AF characters of the four signals. As shown in Figure 8, carrying more communication bits, the main energy of the Chirp-MSK signal has more expansion and diffusion, while the main energy of the JRC signal also tends to be on the straight line $f=u \cdot t$. So the JRC signal has excellent performance among the three signals in terms of time-frequency character. 


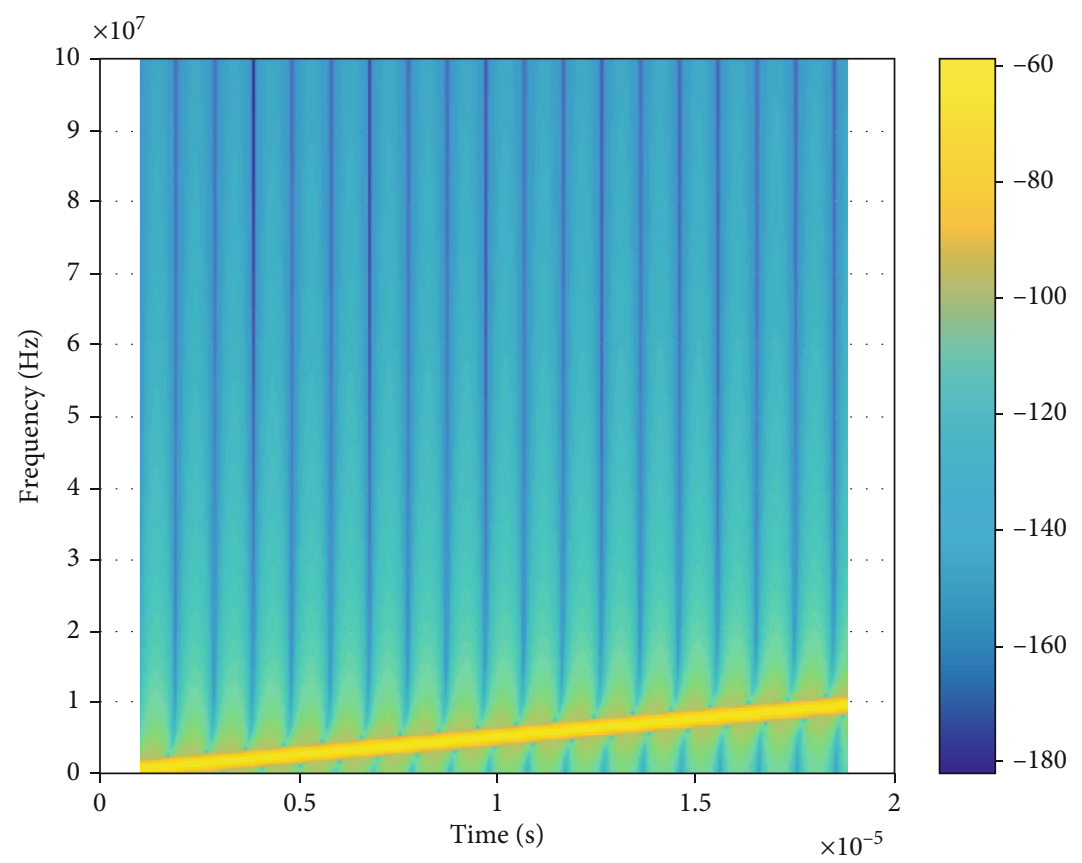

(a)

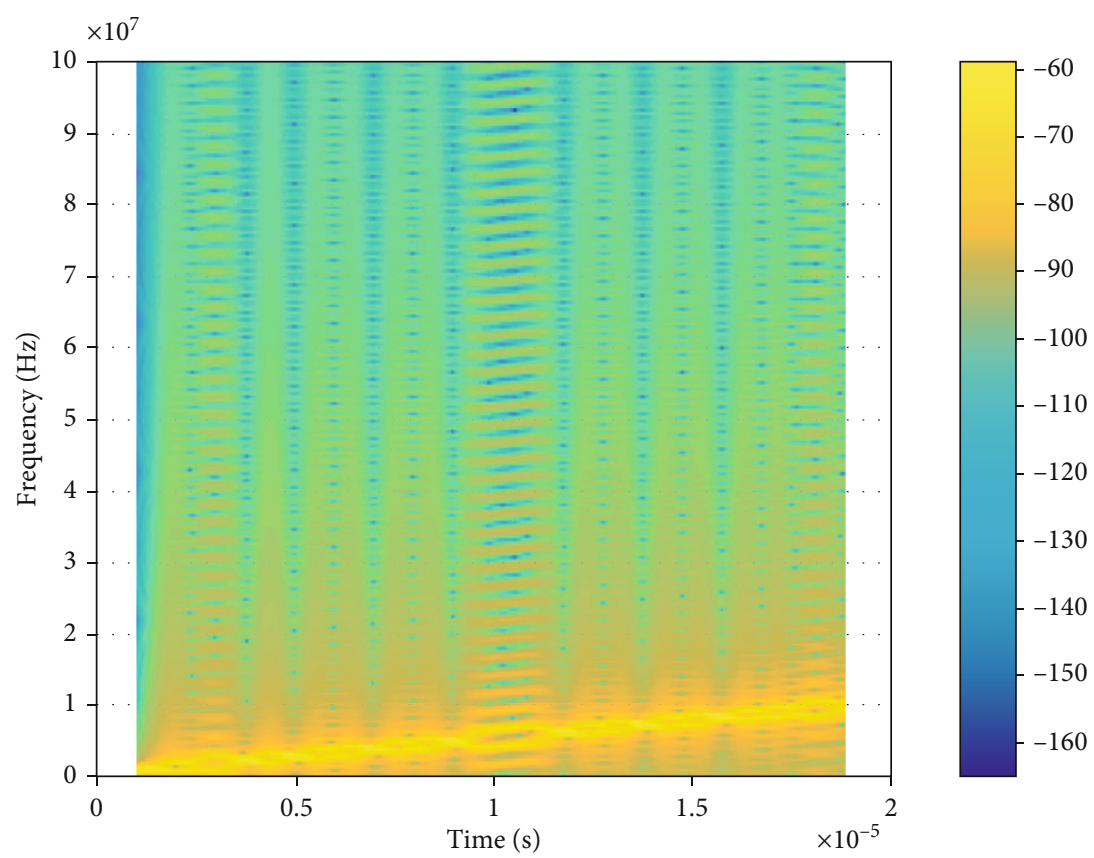

(b)

Figure 7: Continued. 


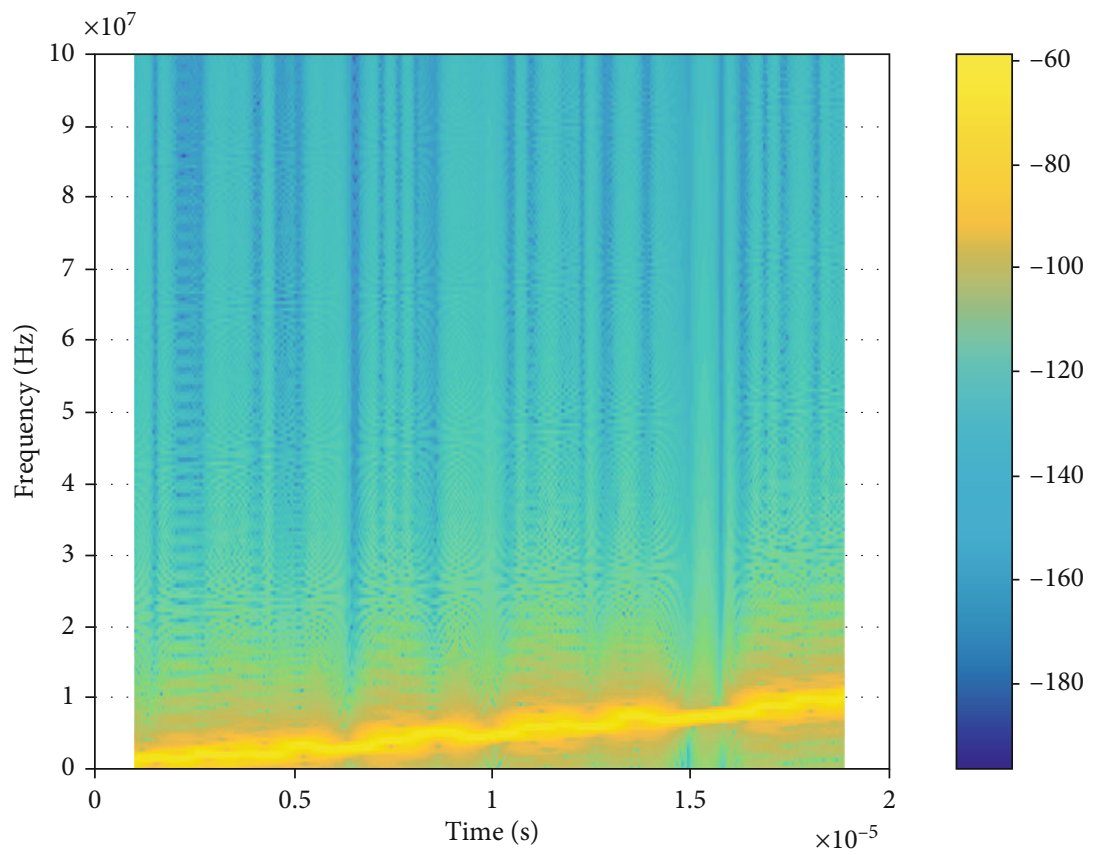

(c)

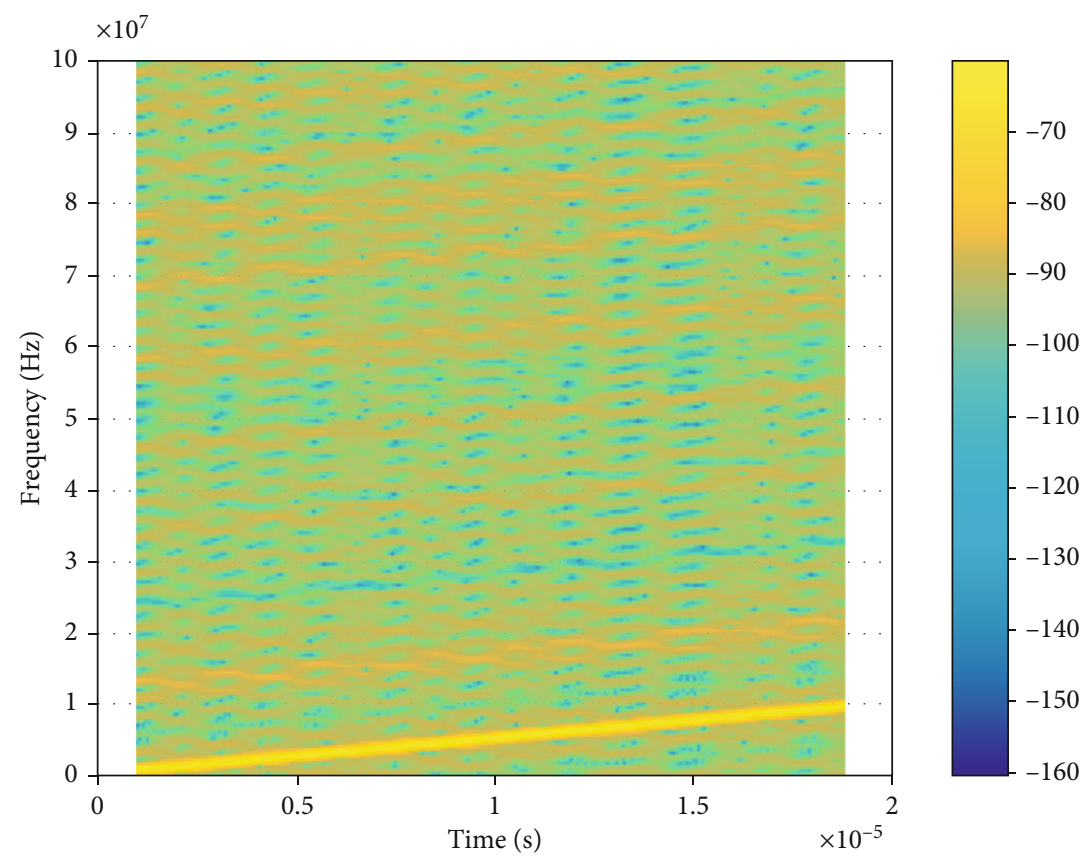

(d)

Figure 7: Comparison of time-frequency analysis graphs for different signals: (a) Chirp signal; (b) Chirp-BPSK signal with 50-bit data; (c) Chirp-MSK signal [13] with 50-bit data; (d) JRC signal with 50-bit data.

5.4. Radar Performance. Now, we focus on the radar detection performance, mainly investigating the AF characters of signals. The AFs of Chirp signal, Chirp-BPSK signal, ChirpMSK signal, and JRC signal are simulated; the top view results of AF are shown in Figures 9 and 10. As shown in Figure 9, the AF of the Chirp signal is a standard bevel blade. Because of the embedded communication data, the phase shift leads to the sharp deterioration of the AF of the Chirp-BPSK signal and Chirp-MSK signal, while the AF of the JRC signal retains the characteristics of the Chirp signal. When the Chirp-BPSK signal and Chirp-MSK signal are used for radar detection, the radar performance decreases significantly, the range detection performance becomes worse, and there are more fluctuations in the Doppler domain. After 


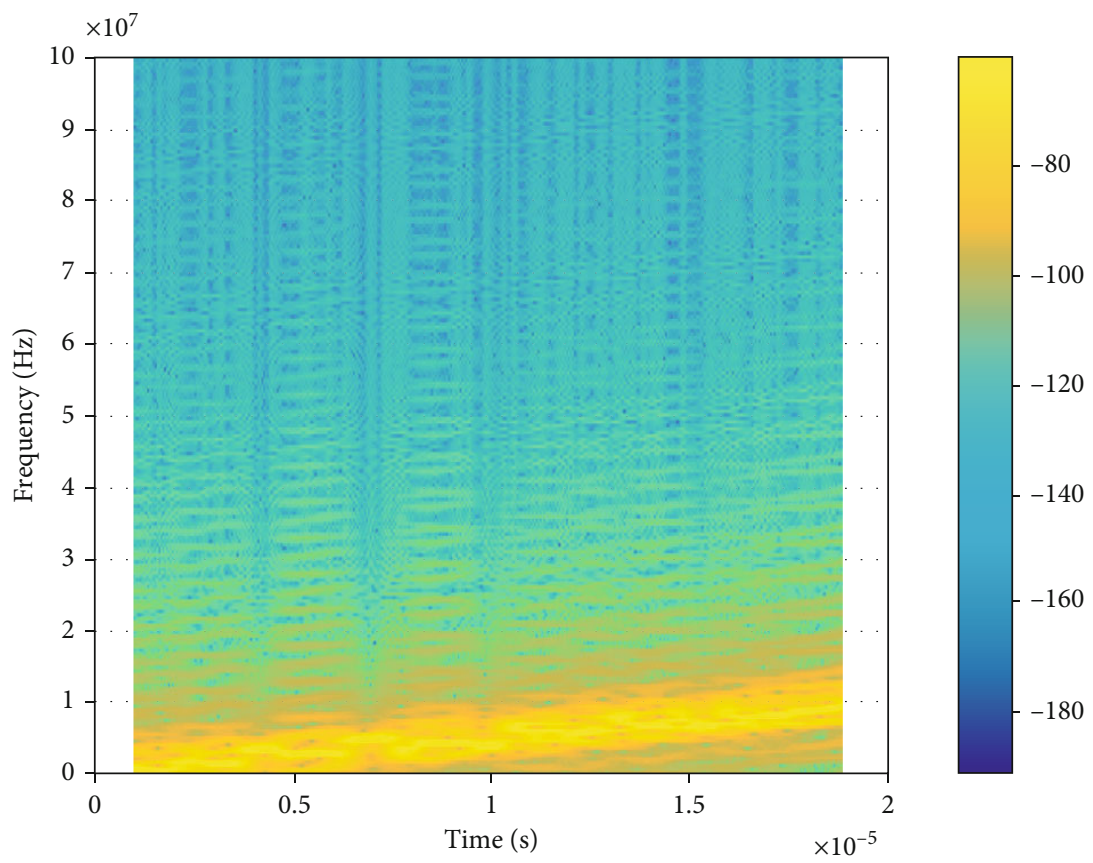

(a)

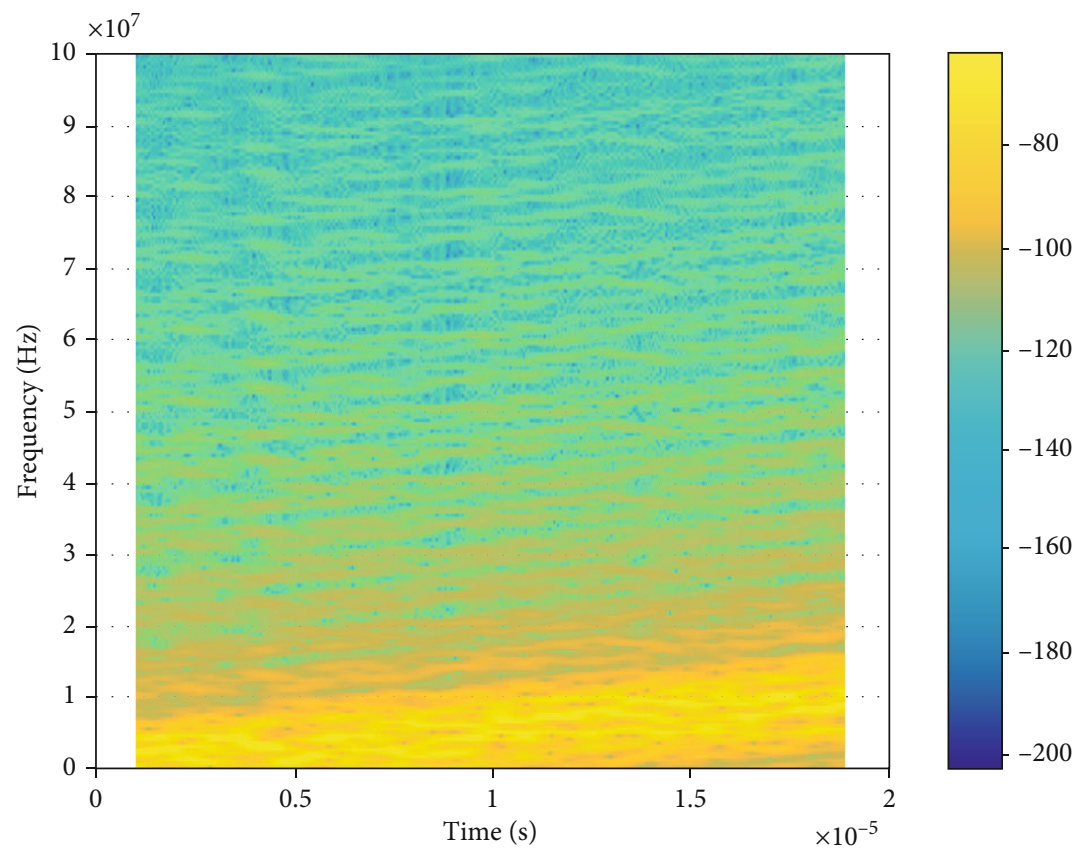

(b)

Figure 8: Continued. 


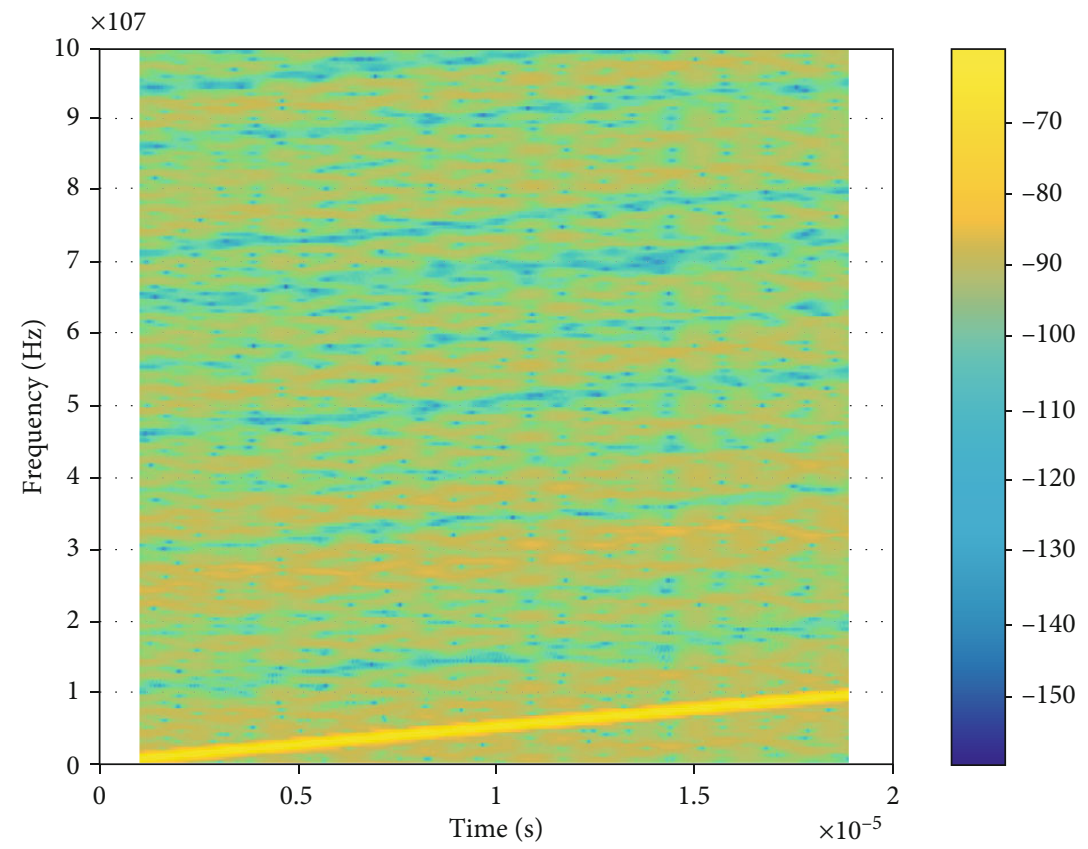

(c)

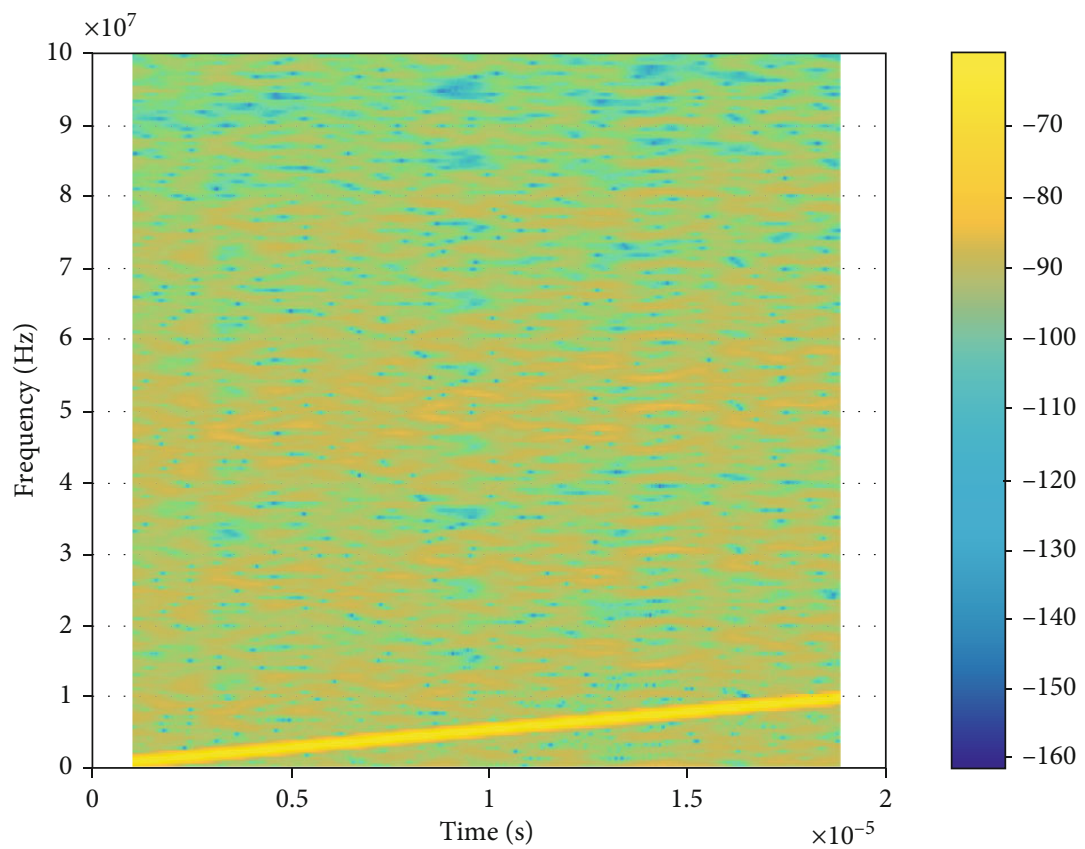

(d)

FIGURE 8: Comparison of time-frequency analysis graphs for Chirp-MSK signal [13] and JRC signal: (a) Chirp-MSK signal with 100-bit data; (b) Chirp-MSK signal with 200-bit data. (c) JRC signal with 100 bits data. (d) JRC signal with 200-bit data.

the JRC signal is designed with communication information, by improving the BPSK modulation and spread spectrum technology, the phase shift is reduced and the loss of BER is compensated.

We consider the AF characteristics of the JRC signal in depth; the AFs of JRC and Chirp-MSK signal are simulated when the communication rate varies, while assuming the other parameters remain unchanged. When the communication rate is enhanced, the simulation results of the ChirpMSK signal and JRC signal are shown in Figure 10. As shown in Figure 10, the top view of AF of the JRC signal retains the characteristics of the Chirp signal pulse while the top view of AF of Chirp-MSK signal has more expansion and diffusion. Because the phase shift is tiny, when the communication rate 


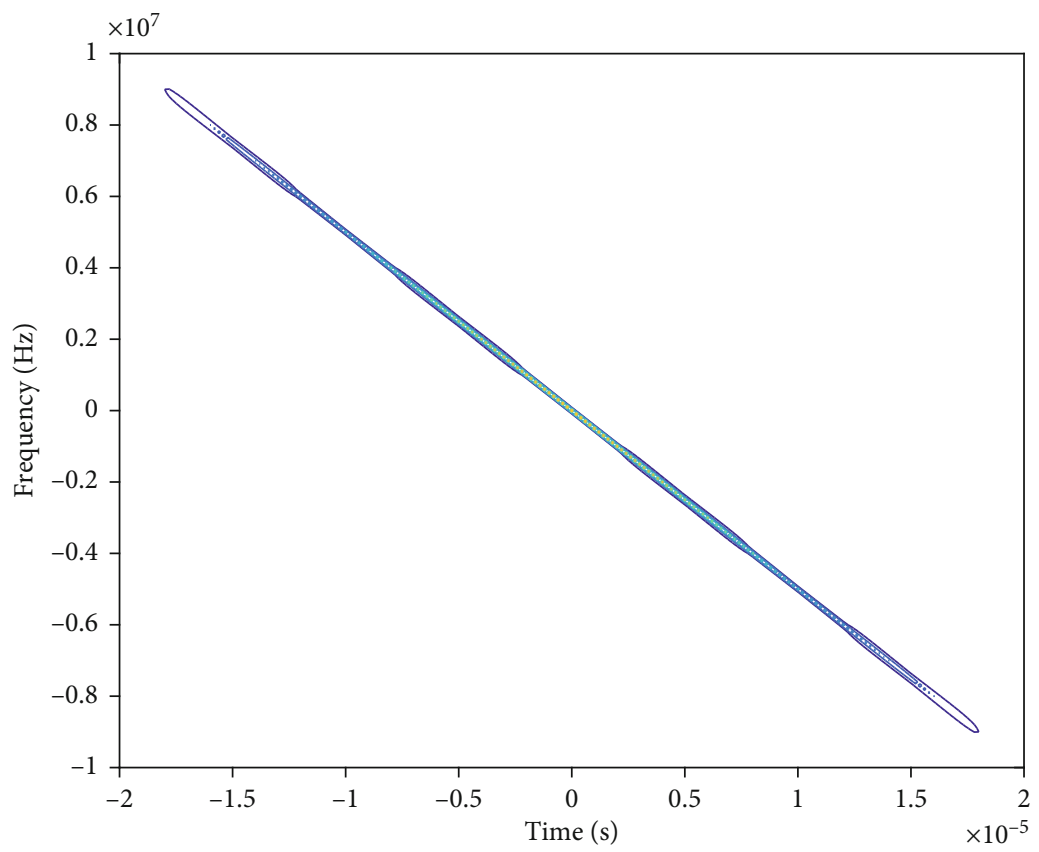

(a)

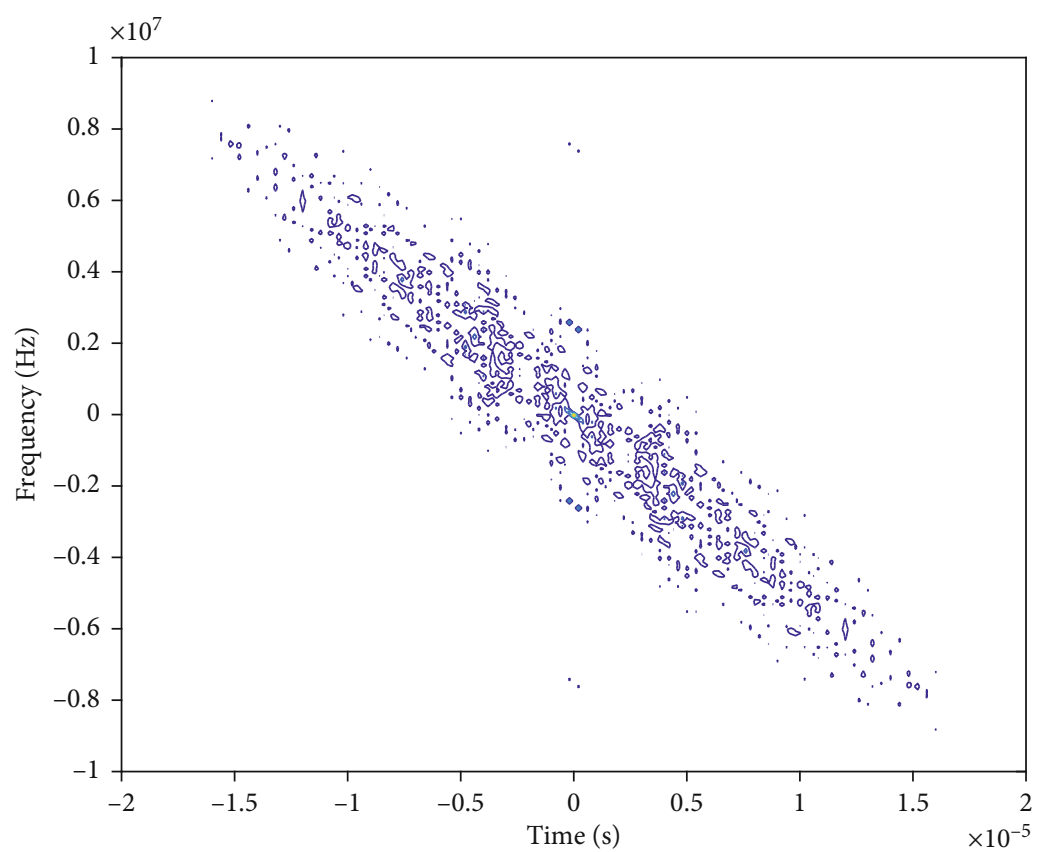

(b)

Figure 9: Continued. 


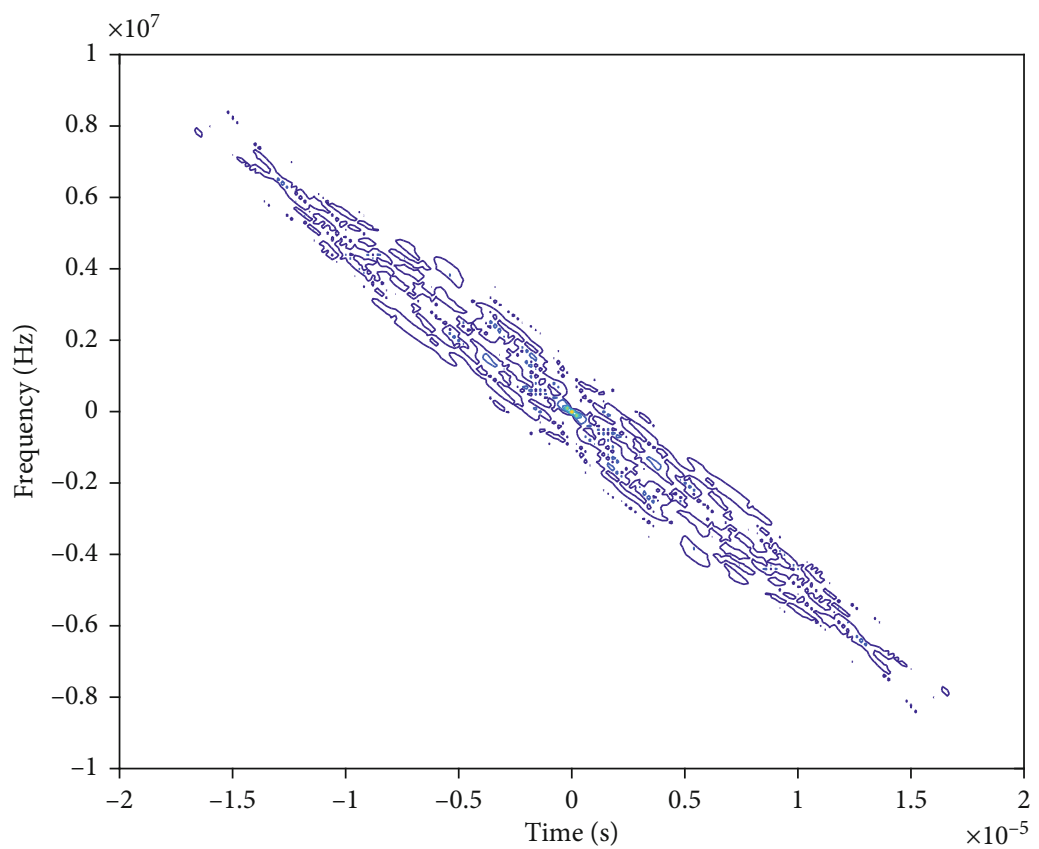

(c)

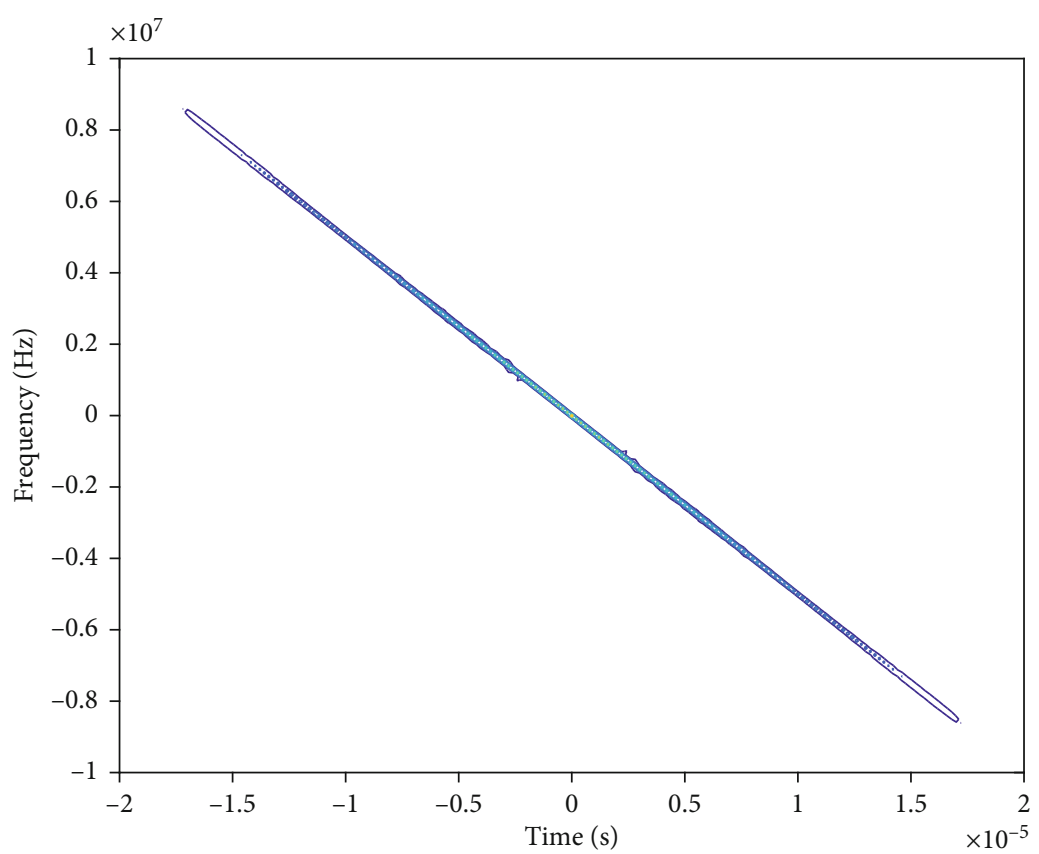

(d)

Figure 9: Top view of AF for different signals: (a) Chirp signal; (b) Chirp-BPSK signal with 50 bit data; (c) Chirp-MSK signal [13] with 50 bit data; (d) JRC signal with 50 bit data.

of the JRC signal increases, the influence on the delay characteristics and Doppler characteristics can be ignored, and the overall detection performance will not be affected. The performance analysis results in Section 4 and Section 5.3 are verified.

In this paper, the JRC signal can effectively solve the problem of low communication throughput of the integration signal of radar and communication. Finally, it is clear that our proposed method is better than the Chirp-BPSK sig- nal and Chirp-MSK signal in joint radar-communication scenarios.

\section{Conclusions}

Prior JRC signals bring many advantages, but also drawback the radar detection performance. In this paper, a novel JRC method based on hybrid CSSC and improved BPSK modulation has been proposed to solve these problems. Using a 


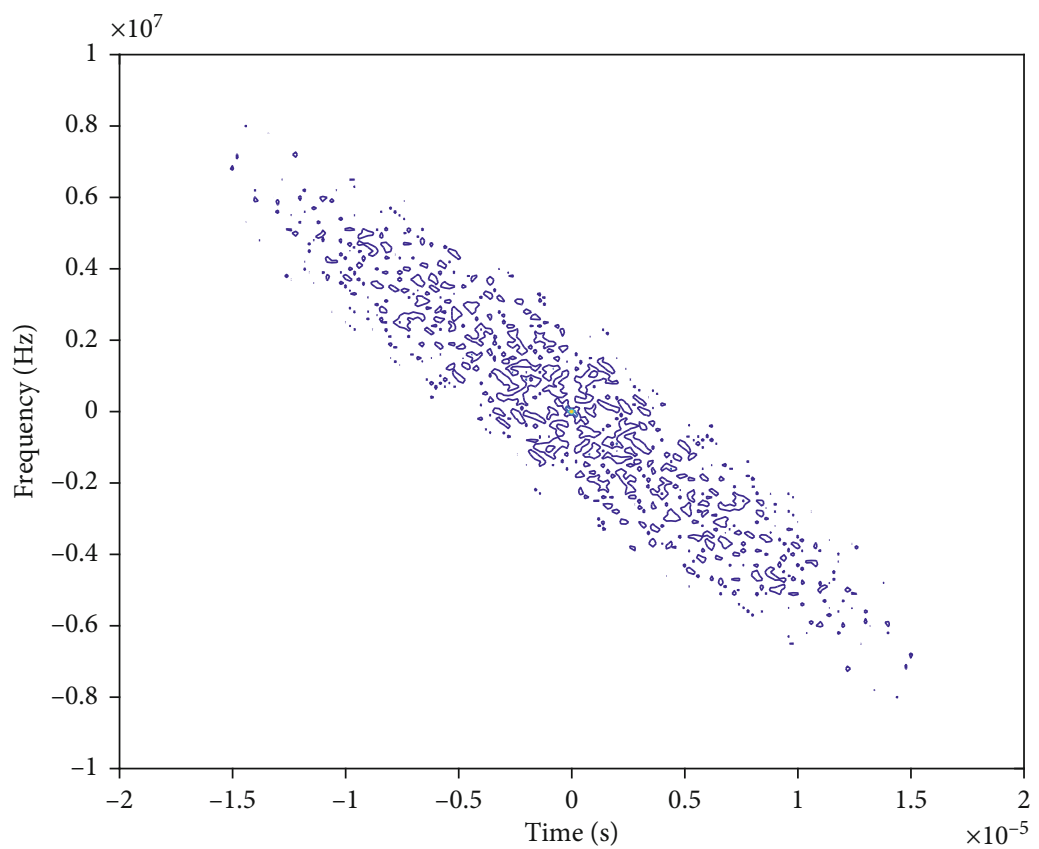

(a)

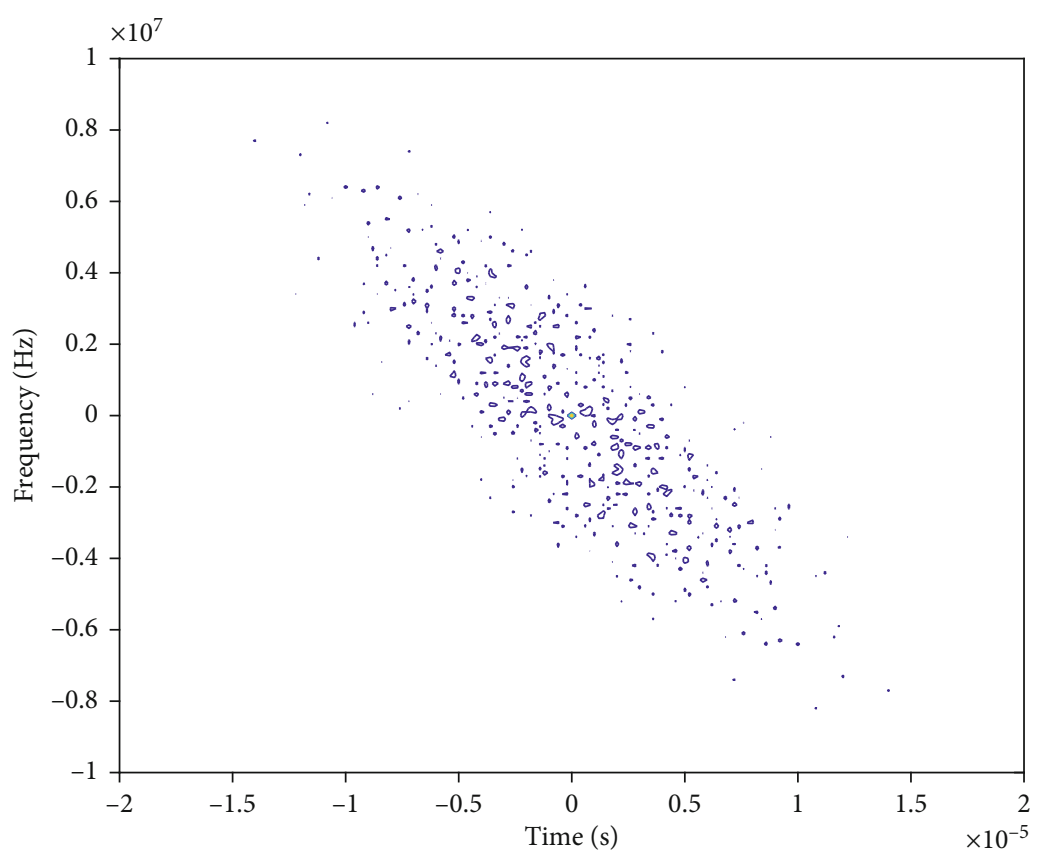

(b)

Figure 10: Continued. 


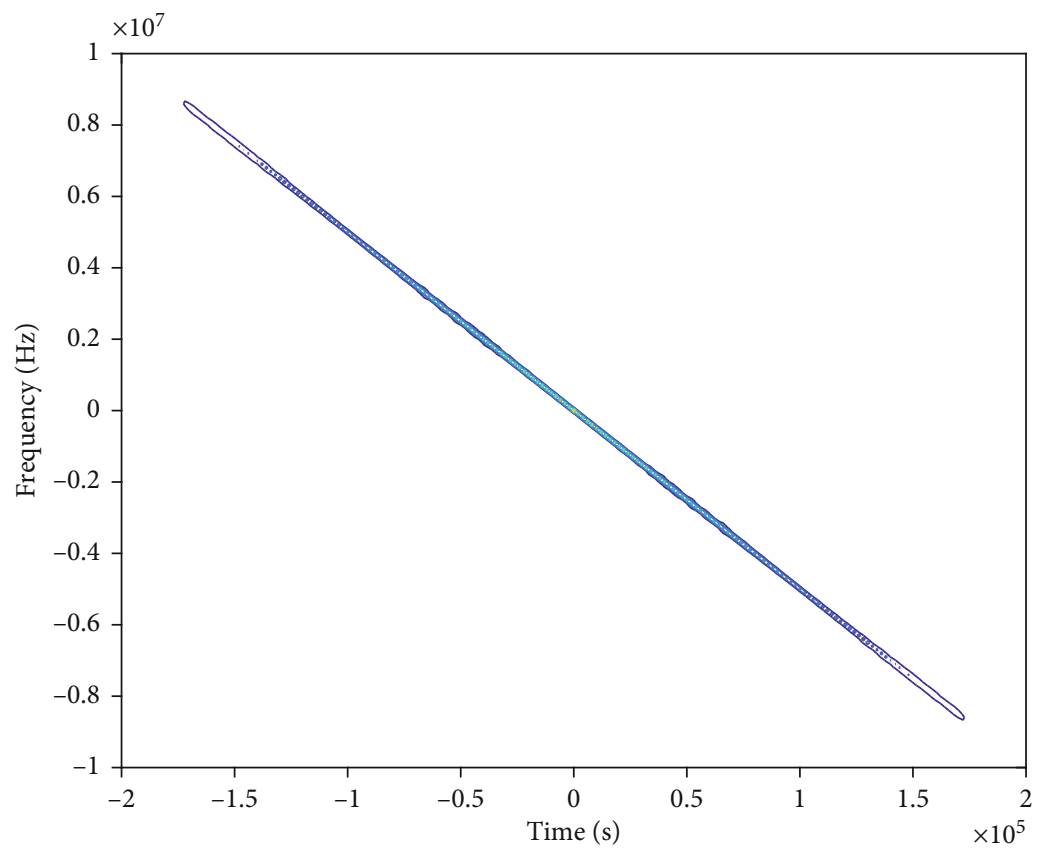

(c)

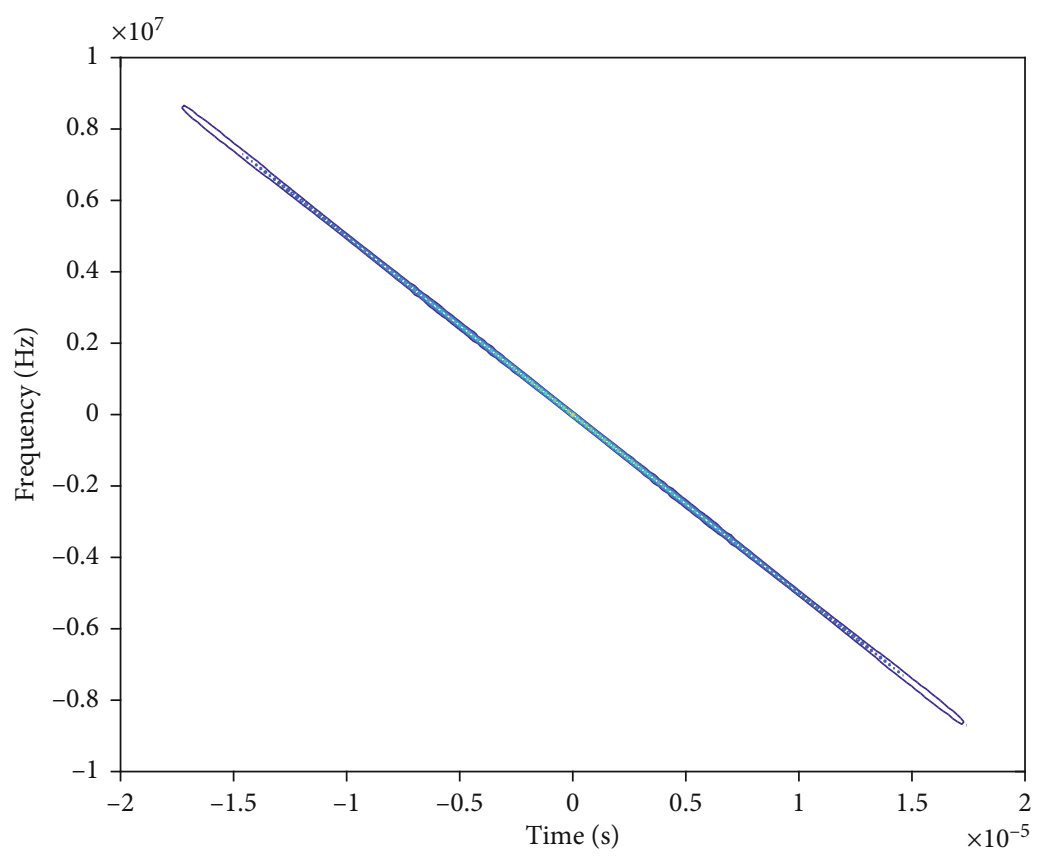

(d)

FIgURE 10: Top view of AF for Chirp-MSK signal [13] and JRC with different data: (a) Chirp-MSK signal with 100 bits; (b) Chirp-MSK signal with 200 bits; (c) JRC signal with 100 bits; (d) JRC signal with 200 bits.

hybrid CSSC structure, the proposed scheme improves the anti-interference and increases the communication data. In the performance analysis of the JRC signal, it is known that the improved phase modulation mode and pseudorandom characteristics of hybrid CSSC eliminate the problem of phase shift and accumulation. The JRC signal with excellent spectrum characteristics and AF characteristics, whose performance is better than the performance of the Chirp-BPSK signal and Chirp-MSK signal. Through computer simulation, compared with Chirp-BPSK signal and Chirp-MSK signal, our proposed JRC signal can transmit communication data with tiny spectral distortion, high range-Doppler resolution, and can effectively solve the problem of low communication rate. 


\section{Appendix}

\section{A. Spectrum Equation of Chirp-BPSK}

In the following, the spectrum equation of Chirp-BPSK is derived as:

$$
\begin{aligned}
S_{\text {Chirp-BPSK }}(f)= & \int_{-\infty}^{\infty} s_{\text {Chirp-BPSK }}(t) \cdot \exp (-j \cdot 2 \cdot \pi \cdot f \cdot t) d t \\
= & \int_{-\infty}^{\infty} \sum_{i=1}^{N} u\left(t-i T_{b}\right) \cdot \exp \left(j \cdot \pi \cdot u \cdot t^{2}+j \theta_{i}\right) \\
& \cdot \exp (-j \cdot 2 \cdot \pi \cdot f \cdot t) d t \\
= & \sum_{i=1}^{N} \int_{-\infty}^{\infty} u\left(t-i T_{b}\right) \cdot \exp \left(j \cdot \pi \cdot u \cdot t^{2}+j \theta_{i}\right) \\
& \cdot \exp (-j \cdot 2 \cdot \pi \cdot f \cdot t) d t \\
= & \sum_{i=1}^{N} \int_{(i-1) T_{b}}^{T_{b}} \exp \left(j \cdot \pi \cdot u \cdot t^{2}+j \cdot \theta_{i}-j \cdot 2 \cdot \pi \cdot f \cdot t\right) d t \\
= & \sum_{i=1}^{N} \exp \left(j \cdot \theta_{i}\right) \int_{(i-1) T_{b}}^{T_{b}} \exp \left(j \cdot \pi \cdot u \cdot t^{2}-j \cdot 2 \cdot \pi \cdot f \cdot t\right) d t \\
= & \sum_{i=1}^{N} \exp \left(j \cdot \theta_{i}\right) \int_{(i-1) T_{b}}^{T_{b}} \exp \left(j \cdot \pi \cdot u \cdot\left(t^{2}-\frac{2 \cdot f \cdot t}{u}\right) d t\right. \\
= & \sum_{i=1}^{N} \exp \left(j \cdot \theta_{i}\right) \int_{(i-1) T_{b}}^{T_{b}} \exp \left(j \cdot \pi \cdot u \cdot \left(t^{2}-\frac{2 \cdot f \cdot t}{u}\right.\right. \\
& \left.+\left(\frac{f}{u}\right)^{2}-\left(\frac{f}{u}\right)^{2}\right) d t=\sum_{i=1}^{N} \exp \left(j \cdot \theta_{i}-j \cdot \pi \cdot u \cdot\left(\frac{f}{u}\right)^{2}\right) \\
& \cdot \int_{(i-1) T_{b}}^{T_{b}} \exp \left(j \cdot \pi \cdot u \cdot\left(t^{2}-\frac{2 \cdot f \cdot t}{u}+\left(\frac{f}{u}\right)^{2}\right)^{2} d t\right. \\
= & \sum_{i=1}^{N} \exp \left(j \cdot \theta_{i}-j \cdot \pi \cdot \frac{f^{2}}{u}\right)^{T_{b}}(i-1) T_{b} \\
& \exp \left(j \cdot \pi \cdot u \cdot\left(t-\frac{f}{u}\right)^{2}\right) d t . \\
& \\
& \\
&
\end{aligned}
$$

We assume $\left(v^{2} / 2\right)=u \cdot(t-(f / u))^{2}, \quad$ (A.1) can be expressed as:

$$
\begin{aligned}
S_{\text {Chirp-BPSK }}(f)= & \frac{1}{\sqrt{2 \cdot u}} \sum_{i=1}^{N} \exp \left(j \cdot \theta_{i}-j \cdot \pi \cdot \frac{f^{2}}{u}\right) \\
& \cdot \int_{\sqrt{2 u}\left((i-1) T_{b}-\frac{f}{u}\right)}^{\sqrt{2 u}\left(i \cdot T_{b}-\frac{f}{u}\right)} \exp \left(j \cdot \pi \cdot u \cdot \frac{v^{2}}{2}\right) d t,
\end{aligned}
$$

where $x_{3}=\sqrt{2 \cdot u}\left((i-1) T_{b}-f / u\right), x_{4}=\sqrt{2 \cdot u}\left(i \cdot T_{b}-f / u\right)$, and $\theta_{i}= \pm \pi / 2$, so (A.1) can be expressed as:

$$
\begin{aligned}
S_{\text {Chirp-BPSK }}(f)= & \frac{1}{\sqrt{2 \cdot u}} \sum_{i=1}^{N} \exp \left(j \cdot \theta_{i}-j \cdot \pi \cdot \frac{f^{2}}{u}\right) \\
& \cdot\left[\left(C\left(x_{4}\right)-C\left(x_{3}\right)\right)+j \cdot\left(S\left(x_{4}\right)-S\left(x_{3}\right)\right)\right] .
\end{aligned}
$$

\section{B. Equations of Time-Frequency Analysis}

In the section, the time-frequency analysis equations are derived. The Chirp-BPSK waveform with $N$ bits is defined as:

$$
s_{\text {Chirp-BPSK }}(t)=\sum_{i}^{N} u\left(t-i T_{b}\right) \cdot \exp \left(j \cdot\left(\pi \cdot u \cdot t^{2}+\theta_{i}\right)\right) .
$$

For the $i$-th bit, the Chirp-BPSK can be written as [10-12]:

$$
s_{\mathrm{i}}(t)=u\left(t-i T_{b}\right) \cdot \exp \left(j \cdot \pi\left(u \cdot t^{2}+\frac{t \cdot b_{i}}{2 \cdot T_{b}}\right)\right),
$$

The Chirp-BPSK with $N$ bits data is rewritten as:

$s_{\text {Chirp-BPSK }}(t)=\sum_{i}^{N} u\left(t-i T_{b}\right) \cdot \exp \left(j \cdot \pi \cdot\left(u \cdot t^{2}+\frac{t \cdot b_{i}}{2 \cdot T_{b}}\right)\right)$.

The STFT function of Chirp-BPSK is derived as:

$$
\begin{aligned}
\operatorname{STFT}_{y}(t, f)= & \int_{-\infty}^{\infty} \sum_{i=1}^{N} u\left(\tau-i T_{b}\right) \cdot \exp \left(j \cdot \pi\left(u \cdot \tau^{2}+\tau \cdot b_{i} /\left(2 \cdot T_{b}\right)\right)\right. \\
& \cdot w^{*}(\tau-t) \cdot \exp (-j \cdot 2 \cdot \pi \cdot f \cdot \tau) d \tau \\
= & \int_{-\infty}^{\infty} \sum_{i=1}^{N} u\left(\tau-i T_{b}\right) \cdot \exp \left(j \cdot \pi \left(u \cdot \tau^{2}+u \cdot t^{2}\right.\right. \\
& \left.+u \cdot 2 \cdot t \cdot \tau-u \cdot 2 \cdot t \cdot \tau-u \cdot t^{2}\right) \\
& \cdot \exp \left(-j \cdot \pi \cdot \frac{\tau \cdot b_{i}}{2 \cdot T_{b}}\right) \cdot w^{*}(\tau-t) \\
& \cdot \exp (-j \cdot 2 \cdot \pi \cdot f \cdot \tau) d \tau \\
= & \exp \left(-j \cdot \pi \cdot u \cdot t^{2}\right) \int_{-\infty}^{\infty} \sum_{i=1}^{N} u\left(\tau-i T_{b}\right) \\
& \cdot \exp \left(-j \cdot \pi \cdot \frac{\tau \cdot b_{i}}{2 \cdot T_{b}}\right) \cdot \exp \left(j \cdot \pi \cdot u \cdot(\tau-t)^{2}\right) \\
& \cdot \exp (j \cdot \pi \cdot u \cdot 2 \cdot t \cdot \tau) \cdot w(\tau-t) \\
& \cdot \exp (-j \cdot 2 \cdot \pi \cdot f \cdot \tau) d \tau \\
= & \left.\exp \left(-j \cdot \pi \cdot u \cdot t^{2}\right) \sum_{i=1}^{N} u\left(\tau-i T_{b}\right)\right\}_{-\infty}^{\infty} \\
& \cdot \exp \left(-j \cdot \pi \cdot \frac{\tau \cdot b_{i}}{2 \cdot T_{b}}\right) \cdot \exp \left(j \cdot \pi \cdot u \cdot(\tau-t)^{2}\right) \\
& \cdot \exp (j \cdot \pi \cdot u \cdot 2 \cdot t \cdot \tau) \cdot w(\tau-t) \\
& \cdot \exp (-j \cdot 2 \cdot \pi \cdot f \cdot \tau) d \tau,
\end{aligned}
$$

We assume $\mathrm{w}(\tau)$ is the Gauss window function, and we define the function: 


$$
B(f)=\int_{-\infty}^{\infty} \exp \left(j \cdot \pi \cdot u \cdot \tau^{2}\right) \cdot w(\tau) \cdot \exp (-j \cdot 2 \pi \cdot f \cdot \tau) d \tau
$$

The $\mathrm{B}(f)$ can be expressed as Fourier transmit of windowed Chirp signal. The above equations are time shift and frequency shift operations for the windowed Chirp signal.

The time shift and frequency shift for Fourier formulas are as follows:

$$
\left\{\begin{array}{l}
s(t) \leftrightarrow S(f), \\
s\left(t-t_{0}\right) \leftrightarrow S(f) \cdot \exp \left(-j \cdot 2 \pi \cdot f \cdot t_{0}\right), \\
s(t) \exp \left(j \cdot 2 \pi \cdot f_{0} \cdot t\right) \leftrightarrow S\left(f-f_{0}\right) .
\end{array}\right.
$$

$\operatorname{The~} \operatorname{STFT}_{y}(t, f)$ is derived as:

$$
\begin{aligned}
\operatorname{STFT}_{y}(t, f)= & \exp (-j \cdot \pi \cdot u \cdot t \cdot(t+2 f)) \sum_{i=1}^{N} u\left(\tau-i T_{b}\right) \int_{-\infty}^{\infty} \\
& \cdot \exp \left(j \cdot \pi \cdot u \cdot(\tau-t)^{2}\right) \\
& \cdot \exp \left(j \cdot \pi \cdot 2 \cdot \tau\left(u \cdot t+\frac{b_{i}}{4 T_{b}}\right)\right) \\
& \cdot w(\tau-t) \cdot \exp (-j \cdot 2 \cdot \pi \cdot f \cdot(\tau-t)) d \tau \\
= & \exp (-j \cdot \pi \cdot u \cdot t \cdot(t+2 f)) \sum_{i=1}^{N} u\left(\tau-i T_{b}\right) \\
& \cdot B\left(f-\left(u \cdot t+\frac{b_{i}}{4 T_{b}}\right)\right) \\
& \cdot \exp \left(-j \cdot 2 \cdot \pi \cdot t \cdot\left(f-\left(u \cdot t+\frac{b_{i}}{4 T_{b}}\right)\right)\right) .
\end{aligned}
$$

The Gauss window function is defined as $w(t)=$ $\exp \left(-t^{2} / 2 \cdot \sigma^{2}\right)$, while $\sigma$ is constant. We get the equation:

$$
\begin{aligned}
B(f)= & \int_{-\infty}^{\infty} \exp \left(j \cdot \pi \cdot u \cdot \tau^{2}\right) \cdot \exp \left(\frac{-\tau^{2}}{2 \cdot \sigma^{2}}\right) \\
& \cdot \exp (-j \cdot 2 \pi \cdot f \cdot \tau) d \tau .
\end{aligned}
$$

The phase of $B(f)$ is

$$
\Phi=j \cdot \pi \cdot u \cdot \tau^{2}-\frac{\tau^{2}}{2 \cdot \sigma^{2}}-j \cdot 2 \pi \cdot f \cdot \tau .
$$

We get the stationary phase for letting the first derivative of $\Phi$ equal null $[13,14]$ :

$$
\begin{gathered}
\frac{d \Phi}{d \tau}=2 \cdot j \cdot \pi \cdot u \cdot \tau-\frac{\tau}{\sigma^{2}}-j \cdot 2 \pi \cdot f=0, \\
\tau^{*}=\frac{j \cdot 2 \pi \cdot f}{2 \cdot j \cdot \pi \cdot u-1 / \sigma^{2}} .
\end{gathered}
$$

Substitute (B.11) into (B.8), we get equation (B.12).

$$
\begin{aligned}
B(f)= & \frac{2 \cdot \sqrt{\pi}}{\sqrt{(2 \cdot \pi \cdot u)^{2}+\left(1 / \sigma^{4}\right)}} \cdot \exp \left(-\frac{1}{2} \cdot \frac{(2 \cdot j \cdot \pi \cdot f)^{2}}{2 \cdot j \cdot \pi \cdot u-1 / \sigma^{2}}\right) \\
= & \frac{2 \cdot \sigma^{2} \cdot \sqrt{\pi}}{\sqrt{\left(2 \cdot \pi \cdot \sigma^{2} \cdot u\right)^{2}+1}} \\
& \cdot \exp \left(-j \cdot \frac{\pi \cdot u \cdot(2 \cdot \pi \cdot f)^{2}}{\left(2 \cdot j \cdot \pi \cdot \sigma^{2} \cdot u\right)^{2}+1}+\frac{2 \cdot(\sigma \cdot \pi \cdot f)^{2}}{\left(2 \cdot j \cdot \pi \cdot \sigma^{2} \cdot u\right)^{2}+1}\right) .
\end{aligned}
$$

Substitute (B.12) into (B.7), we get the equations (B.13).

$$
\begin{aligned}
\operatorname{STFT}_{y}(t, f)= & \frac{2 \cdot \sigma^{2} \cdot \sqrt{\pi}}{\sqrt{\left(2 \cdot \pi \cdot u \cdot \sigma^{2}\right)^{2}+1}} \exp (-j \cdot \pi \cdot u \cdot t \cdot(t+2 f)) \\
& \cdot \sum_{i=1}^{N} u\left(\tau-i T_{b}\right) \exp \left(-j \cdot \pi \cdot 2 \cdot t\left(f-\left(u \cdot t+\frac{b_{i}}{4 T_{b}}\right)\right)\right. \\
& \cdot \exp \left[\frac{2 \cdot\left(\sigma \cdot \pi\left(f-\left(u \cdot t+\left(b_{i} / 4 T_{b}\right)\right)\right)\right)^{2}}{\left(2 \cdot \pi \cdot \sigma^{2} \cdot u\right)^{2}+1}\right. \\
& \left.-\frac{j \cdot \pi \cdot u \cdot\left(2 \cdot \pi\left(f-\left(u \cdot t+\left(b_{i} / 4 T_{b}\right)\right)\right)\right)^{2}}{\left(2 \cdot \pi \cdot \sigma^{2} \cdot u\right)^{2}+1}\right] .
\end{aligned}
$$

The spectrogram for the Chirp-BPSK signal is derived as [13]:

$S_{p}(t, f)=A^{2} \exp \left\{\frac{2 \cdot\left[\pi \cdot \sigma\left(f-\left(u \cdot t+\sum_{i=1}^{N} u\left(t-i T_{b}\right) \cdot b_{i} / 4 T_{b}\right)\right]^{2}\right.}{\left(2 \cdot \pi \cdot \sigma^{2} \cdot \mu\right)^{2}+1}\right\}$.

where $A^{2}$ is the amplitude of the spectrogram.

\section{Data Availability}

I have not included a data availability statement in my manuscript.

\section{Conflicts of Interest}

The authors declare no conflict of interest.

\section{Acknowledgments}

This work was supported by the Foundations of Beijing Jiaotong University of China under Grants W15H100020 and W16GY500010.

\section{References}

[1] B. Hong, W.-Q. Wang, and C.-C. Liu, "Ergodic interference alignment for spectrum sharing radar-communication systems," IEEE Transactions on Vehicular Technology, vol. 68, no. 10, pp. 9785-9796, 2019. 
[2] B. Paul, A. R. Chiriyath, and D. W. Bliss, "Survey of RF communications and sensing convergence research," IEEE Access, vol. 5, pp. 252-270, 2017.

[3] M. Arik and O. B. Akan, "Capacity analysis for joint radarcommunication capable coherent MIMO radars," Physical Communication, vol. 40, article 101062, 2020.

[4] B. Kong, Y. Wang, H. Leung, X. Deng, H. Zhou, and F. Zhou, "Sparse representation based range-Doppler processing for integrated OFDM radar-communication networks," International Journal of Antennas and Propagation, vol. 2017, Article ID 6528956, 12 pages, 2017.

[5] A. Gameiro, D. Castanheira, J. Sanson, and P. P. Monteiro, "Research challenges, trends and applications for future joint radar communications systems," Wireless Personal Communications, vol. 100, no. 1, pp. 81-96, 2018.

[6] S. Quan, W. Qian, J. Guq, and V. Zhang, "Radar-communication integration: an overview," in The 7th IEEE/International Conference on Advanced Infocomm Technology, pp. 98-103, Fuzhou, November 2014.

[7] J. Zhuang, Q. Ye, Q. Tan, and A. H. Ali, "Low-complexity variable loading for robust adaptive beamforming," Electronics Letters, vol. 52, no. 5, pp. 338-340, 2016.

[8] S. D. Blunt and E. L. Mokole, "Overview of radar waveform diversity," IEEE Aerospace and Electronic Systems Magazine, vol. 31, no. 11, pp. 2-42, 2016.

[9] M. Roberton and E. R. Brown, "Integrated radar and communications based on chirped spread-spectrum techniques," in IEEE MTT-S International Microwave Symposium Digest, 2003, vol. 1, pp. 611-614, Philadelphia, PA, USA, 2003.

[10] L. Jiang, S. Yan, Y. Wu, and X. Ma, "Sonar detection performance with LFM-BPSK combined waveforms," in OCEANS 2016 - Shanghai, pp. 1-4, Shanghai, April 2016.

[11] Z. Zhao and D. Jiang, "A novel integrated radar and communication waveform based on LFM signal," in 2015 IEEE 5th International Conference on Electronics Information and Emergency Communication, pp. 219-223, Beijing, May 2015.

[12] M. Nowak, M. Wicks, Z. Zhang, and Z. Wu, "Co-designed radar-communication using linear frequency modulation waveform," IEEE Aerospace and Electronic Systems Magazine, vol. 31, no. 10, pp. 28-35, 2016.

[13] L. Zhipeng, C. Xingbo, W. Xiaomo, S. Xu, and F. Yuan, "Communication analysis of integrated waveform based on LFM and MSK," in IET International Radar Conference 2015, pp. 1-5, Hangzhou, 2015.

[14] X. Chen, Z. Liu, Y. Liu, and Z. Wang, "Energy leakage analysis of the radar and communication integrated waveform," IET Signal Processing, vol. 12, no. 3, pp. 375-382, 2018.

[15] Y. Ni, Z. Wang, Q. Huang, and M. Zhang, "High throughput rate-shift integrated system for joint radar-communications," IEEE Access, vol. 7, pp. 78228-78238, 2019.

[16] Z. Liu, W. Zhang, and S. Xu, "Implementation on the integrated waveform of radar and communication," in 2013 International Conference on Communications, Circuits and Systems (ICCCAS), pp. 200-204, Chengdu, November 2013.

[17] D. Chen and C. Zhang, "Integrated communication waveform design for bistatic radar," in 2018 IEEE/AIAA 37th digital avionics systems conference (DASC), pp. 1-6, London, September 2018.

[18] M. J. Li, W. Q. Wang, and Z. Zheng, "Communication-embedded OFDM chirp waveform for delay-Doppler radar," IET Radar, Sonar \& Navigation, vol. 12, no. 3, pp. 353-360, 2018.
[19] S. Y. Nusenu, H. Chen, and W. Q. Wang, "OFDM chirp radar for adaptive target detection in low grazing angle," IET Signal Processing, vol. 12, no. 5, pp. 613-619, 2018.

[20] A. Balleri and A. Farina, "Ambiguity function and accuracy of the hyperbolic chirp: comparison with the linear chirp," IET Radar, Sonar \& Navigation, vol. 11, no. 1, pp. 142-153, 2017.

[21] Y. Zhang, Q. Li, L. Huang, C. Pan, and J. Song, "A modified waveform design for radar-communication integration based on LFM-CPM," in 2017 IEEE 85th Vehicular Technology Conference (VTC Spring), pp. 1-5, Sydney, NSW, June 2017.

[22] Y. Zhang, Q. Li, L. Huang, K. Dai, and J. Song, "Waveform design for joint radar-communication with nonideal power amplifier and outband interference," in 2017 IEEE wireless communications and networking conference (WCNC), pp. 16, San Francisco, CA, March 2017.

[23] Q. Li, K. Dai, Y. Zhang, and H. Zhang, "Integrated waveform for a joint radar-communication system with high-speed transmission," IEEE Wireless Communications Letters, vol. 8, no. 4, pp. 1208-1211, 2019.

[24] C. Sahin, J. Jakabosky, P. M. McCormick, J. G. Metcalf, and S. D. Blunt, "A novel approach for embedding communication symbols into physical radar waveforms," in 2017 IEEE Radar Conference (RadarConf), pp. 1498-1503, Seattle, WA, May 2017.

[25] Z. Chaozhu and C. Qiang, "Design of signal-sharing for radar and communication," in Proceedings 2013 International Conference on Mechatronic Sciences, Electric Engineering and Computer (MEC), pp. 1250-1253, Shengyang, December 2013.

[26] C. Shi, F. Wang, M. Sellathurai, J. Zhou, and S. Salous, "Power minimization-based robust OFDM radar waveform design for radar and communication systems in coexistence," IEEE Transactions on Signal Processing, vol. 66, no. 5, pp. 13161330, 2018.

[27] C. Shi, F. Wang, M. Sellathurai, J. Zhou, and S. Salous, "Low probability of intercept-based optimal power allocation scheme for an integrated multistatic radar and communication system," IEEE Systems Journal, vol. 14, no. 1, pp. 983-994, 2020.

[28] C. Shi, F. Wang, S. Salous, and J. Zhou, "Joint subcarrier assignment and power allocation strategy for integrated radar and communications system based on power minimization," IEEE Sensors Journal, vol. 19, no. 23, pp. 11167-11179, 2019.

[29] M. J. Nowak, Z. Zhang, L. LoMonte, M. Wicks, and Z. Wu, "Mixed-modulated linear frequency modulated radar-communications," IET Radar, Sonar \& Navigation, vol. 11, no. 2, pp. 313-320, 2017.

[30] L. Xiao, G. Xuan, and Y. Wu, "Research on an improved chaotic spread spectrum sequence," in 2018 IEEE 3rd International Conference on Cloud Computing and Big Data Analysis (ICCCBDA), pp. 420-423, Chengdu, April 2018.

[31] J. Wang and Y. Wang, "Analysis performance of MC-CDMA communication system based on improved Chebyshev sequence," in 2016 2nd IEEE International Conference on Computer and Communications (ICCC), pp. 2277-2280, Chengdu, October 2016.

[32] C. Feng, T. Wang, X. Zhi, and H. Huan, "An effective tracking loop for chirp spread spectrum communication systems," in 2016 IEEE 13th International Conference on Signal Processing (ICSP), pp. 1196-1201, Chengdu, November 2016.

[33] B. R. Mahafza, Radar systems analysis and design using MATLAB, Publishing House of Electronics Industry, Beijing, China, 3rd edition, 2016. 